\title{
Admissibly Stable Manifolds for a Class of Partial Neutral Functional Differential Equations on a Half-line
}

\author{
Thieu Huy Nguyen* and Xuan Yen Trinh
}

Abstract. For the following class of partial neutral functional differential equations

$$
\left\{\begin{array}{l}
\frac{\partial}{\partial t} F u_{t}=B(t) u(t)+\Phi\left(t, u_{t}\right) \quad t \in(0, \infty), \\
u_{0}=\phi \in \mathcal{C}:=C([-r, 0], X)
\end{array}\right.
$$

we prove the existence of a new type of invariant stable and center-stable manifolds, called admissibly invariant manifolds of $\mathcal{E}$-class for the solutions. The existence of such manifolds is obtained under the conditions that the family of linear partial differential operators $(B(t))_{t \geq 0}$ generates the evolution family $\{U(t, s)\}_{t \geq s \geq 0}$ (on Banach space $X$ ) having an exponential dichotomy or trichotomy on the half-line and the nonlinear delay operator $\Phi$ satisfies the $\varphi$-Lipschitz condition, i.e., $\|\Phi(t, \phi)-\Phi(t, \psi)\| \leq \varphi(t) \| \phi-$ $\psi \|_{\mathcal{C}}$ for $\phi, \psi \in \mathcal{C}$, where $\varphi(t)$ belongs to some admissible function space on the halfline. Our main method is based on Lyapunov-Perrons equations combined with the admissibility of function spaces and fixed point arguments.

\section{Introduction}

Consider the following partial neutral functional differential equation (PNFDE)

$$
\frac{\partial}{\partial t} F u_{t}=B(t) u(t)+\Phi\left(t, u_{t}\right), \quad t>0
$$

with the initial datum $u_{0}=\phi \in \mathcal{C}:=C([-r, 0], X)$, where $X$ is a Banach space, $B(t): D(B) \subset X \rightarrow X$ is a (possibly unbounded) linear operator for every fixed $t \geq 0$ with norm $\|\cdot\|_{D(B)}$ and $\|B(t) x\| \leq \widetilde{K}\|x\|_{D(B)}, x \in D(B)$. That is to say, $B(t)$ has the same domain of definition denoted by $D(B)$ for all $t>0$. Furthermore, $F: \mathcal{C} \rightarrow D(B)$ is a bounded linear operator called a difference operator; $\Phi: \mathbb{R}_{+} \times \mathcal{C} \rightarrow X$ is a continuous nonlinear operator called a delay operator, and $u_{t}$ is the history function defined by $u_{t}(\theta):=u(t+\theta)$ for $\theta \in[-r, 0]$.

Received July 27, 2018; Accepted December 17, 2018.

Communicated by Cheng-Hsiung Hsu.

2010 Mathematics Subject Classification. 34C45, 34G20, 35B40, 37D10.

Key words and phrases. exponential dichotomy, partial neutral functional differential equations, stable and center-stable manifolds, admissibility of function spaces.

${ }^{*}$ Corresponding author. 
The research for invariant manifolds is an important object in the study for longtime behavior of solutions to evolution equations. Early results can be traced back to Hadamard [5], Perron [16, 17], Bogoliubov and Mitropolsky [2,3 for existence of invariant manifolds for differential equations in $\mathbb{R}^{n}$ (ODE). Then, Daleckii and Krein [4 proved the existence of invariant manifolds for solutions to semi-linear equations in Banach spaces with bounded linear operators. Henry [6] extended such results to the case of semi-linear parabolic partial differential equations without delays. Huy [13 showed such existences for general semilinear non-autonomous evolution equations in general Banach spaces with nonlinear terms being $\varphi$-Lipschitz which represent complicated diffusion processes. Moreover, recently in [8], Huy has proved the existence of a new type of invariant manifolds, namely the invariant stable manifolds of admissible classes. Such manifolds consist of solutions' trajectories belonging to wide classes of admissible Banach spaces which can be $L_{p}$-spaces, Lorentz or interpolation spaces.

For the case of delay partial functional differential equations (i.e., the special case of (1.1) when $\left.F u_{t}=u(t)\right)$ Minh and Wu 21] used the Graph-transform method to show the existence of invariant manifolds of solutions to delay partial differential equations (see also 22 and reference therein). Such results have then been extended by Huy and Duoc [14 to the case of $\varphi$-Lipschitz delays.

In case of neutral partial functional differential equations (PNFDE) in the autonomous cases (i.e., $B(t)=B$ and $\Phi(t, \phi)=\Phi(\phi)$ do not depend on $t$ ), some results on existence of invariant manifolds have been obtained by H. Petzeltová and O. J. Staffans [18 and by R. Benkhalti, K. Ezzinbi and S. Fatajou [1]. They obtained such results under the conditions that $B$ generates a hyperbolic analytic semigroup, and $\Phi$ is uniform Lipschitz continuous with a small Lipschitz constant.

Results on existence of invariant manifolds in the non-autonomous case for PNFDE (i.e., $B(t)$ and $\Phi(t, \phi)$ depend on time $t$ ) have been obtained by Huy and Bang under the conditions that the family $(B(t))_{t \geq 0}$ generates the dichotomic or trichotomic evolution family, and the delay term $\Phi$ is $\varphi$-Lipshitz, i.e., $\|\Phi(t, \phi)-\Phi(t, \psi)\| \leq \varphi(t)\|\phi-\psi\|_{\mathcal{C}}$, where $\phi, \psi \in \mathcal{C}$ and $\varphi(t)$ is a real function belonging to certain admissible space.

The purpose of the present paper is to extend the results and methods in [9] combining with the methods in 8 to prove the existence of invariant stable manifolds of admissible classes (see Definition 1.2 below) which are constituted by trajectories of solutions belonging to certain Banach space $\mathcal{E}$ which can be an $L_{p}$-space, a Lorentz space $L_{p, q}$ or some interpolation space. We prove the existence of such manifolds for (1.1) when its linear part $(B(t))_{t \geq 0}$ generates the evolution family having an exponential dichotomy or trichotomy on the half-line, and its nonlinear term is $\varphi$-Lipshitz, i.e., $\|\Phi(t, \phi)-\Phi(t, \psi)\| \leq \varphi(t)\|\phi-\psi\|_{\mathcal{C}}$, where $\phi, \psi \in \mathcal{C}$ and $\varphi(t)$ is a real and positive function which belong to admissible function 
space.

As mentioned in [9], when handling with PNFDE we face a difficult fact that the differential operators do not apply directly to $u(t)$ but to $F u_{t}$, and hence the variationof-constant formula is available only for $F u_{t}$. Therefore, we write $F$ in the form $F=$ $\delta_{0}-\left(\delta_{0}-F\right)$, with Dirac distribution $\delta_{0}$ concentrated at 0 . Furthermore, another difficulty is lying in the fact that the admissibly inertial manifold is constituted by trajectories of the solutions belonging to (rescaledly) general admissible function spaces (see Definitions 3.1 and 3.2 thereafter) which are not necessary $L_{\infty}$-spaces. Therefore, the techniques and methodology used in the paper [9] cannot directly be applied here. Instead, we use the duality arguments together with generalized Hölder inequalities to obtain necessary estimates corresponding to the dichotomy of the evolution family. Then we apply our techniques and results in [8] (see also [10]) of using admissibility of function spaces to construct the solutions of Lyapunov-Perron equation which will be used to derive the existence of admissibly stable manifolds of $\mathcal{E}$-class.

Moreover, using these results and rescaling procedures we prove the existence of centerstable manifolds of $\mathcal{E}$-class for the mild solutions to 1.1 in the case of trichotomic linear parts under the same conditions on the nonlinear delay term $\Phi$ as in the dichotomic case. Our main results are contained in Theorems 2.8 , and 3.3 .

We now recall some notions. Let $X$ is a Banach space (with a norm $\|\cdot\|$ ) and for a given $r>0$ we denote by $\mathcal{C}:=C([-r, 0], X)$ the Banach space of all continuous functions from $[-r, 0]$ into $X$, equipped with the norm $\|\phi\|_{\mathcal{C}}=\sup _{\theta \in[-r, 0]}\|\phi(\theta)\|$ for $\phi \in \mathcal{C}$.

For a continuous function $w:[-r, \infty) \rightarrow X$ the history function $w_{t} \in \mathcal{C}$ is defined by $w_{t}(\theta)=v(t+\theta)$ for all $\theta \in[-r, 0]$.

An evolution family is now defined as follows.

Definition 1.1. A family of bounded linear operators $\{U(t, s)\}_{t \geq s \geq 0}$ on a Banach space $X$ is a (strongly continuous, exponentially bounded) evolution family if

(i) $U(t, t)=\mathrm{Id}$ and $U(t, r) U(r, s)=U(t, s)$ for all $t \geq r \geq s \geq 0$,

(ii) the map $(t, s) \mapsto U(t, s) x$ is continuous for every $x \in X$,

(iii) there are constants $K, c \geq 0$ such that $\|U(t, s) x\| \leq K e^{c(t-s)}\|x\|$ for all $t \geq s \geq 0$ and $x \in X$.

The notion of an evolution family arises naturally from the theory of well-posed, nonautonomous abstract Cauchy problem

$$
\left\{\begin{array}{l}
\frac{d u}{d t}=B(t) u(t) \quad t>s \geq 0 \\
u(s)=x \in X
\end{array}\right.
$$


Roughly speaking, the well-posedness of Problem 1.2 means that there exists an evolution family $\{U(t, s)\}_{t \geq s \geq 0}$ such that the solution of 1.2 is given by $u(t)=U(t, s) u(s)$. Also, in this case we say that the family $(B(t))_{t>0}$ generates the evolution family $\{U(t, s)\}_{t \geq s \geq 0}$. For more details on the notion of evolution families, conditions for the existence of such families and applications to partial differential equations we refer the readers to Pazy 15] (see also Nagel and Nickel [12] for a detailed discussion of well-posedness for nonautonomous abstract Cauchy problems on the whole line $\mathbb{R}$ ).

We then briefly recall some notions on function spaces taken from Massera and Schäffer 11, Räbiger and Schnaubelt [19] and Huy [7, 8].

Definition 1.2. Let $E$ be a Banach function space defined as in [7,8 (see also [1]]) and let $X$ be a Banach space endowed with the norm $\|\cdot\|$. We set

$$
\mathcal{E}:=\mathcal{E}\left(\mathbb{R}_{+}, \mathcal{C}\right)=\left\{f: \mathbb{R}_{+} \rightarrow \mathcal{C}: f \text { is strongly measurable and }\|f(\cdot)\|_{\mathcal{C}} \in E\right\}
$$

(modulo $\lambda$-nullfunctions) endowed with the norm $\|f\|_{\mathcal{E}}=\|\| f(\cdot)\left\|_{\mathcal{C}}\right\|_{E}$. One can easily see that $\mathcal{E}$ is a Banach space. We call it the Banach space corresponding to the Banach function space $E$.

In case $E=L^{\infty}\left(\mathbb{R}_{+}\right)$we denote

$$
\mathcal{E}_{\infty}:=\left\{f: \mathbb{R}_{+} \rightarrow \mathcal{C}: f \text { is strongly measurable and }\|f(\cdot)\|_{\mathcal{C}} \in L^{\infty}\left(\mathbb{R}_{+}\right)\right\}
$$

In order to study the invariant manifolds of $\mathcal{E}$-class for semi-linear evolution equations we need some restrictions on the admissible Banach function spaces and assume the following hypothesis.

Standing Hypothesis 1.3. Throughout this paper we consider the admissible Banach function space $E$ such that its associate space $E^{\prime}$ is also an admissible Banach function space. Moreover, for such an admissible Banach function space $E$ we suppose that $E^{\prime}$ contains an exponentially $E$-invariant function, that is the function $\varphi \geq 0$ having the property that, for any fixed $\nu>0$ the function $h_{\nu}$ defined by

$$
h_{\nu}(t):=\left\|e^{-\nu|t-\cdot|} \varphi(\cdot)\right\|_{E^{\prime}} \quad \text { for } t \geq 0
$$

belongs to $E$.

\section{Exponential dichotomy and stable manifolds}

In order to prove the existence of invariant stable manifolds we need the following notion of exponential dichotomies of the evolution family $\{U(t, s)\}_{t \geq s \geq 0}$. 
Definition 2.1. An evolution family $\{U(t, s)\}_{t \geq s \geq 0}$ on the Banach space $X$ is said to have an exponential dichotomy on $[0, \infty)$ if there exist bounded linear projections $P(t)$, $t \geq 0$, on $X$ and positive constants $N, \nu$ such that

(i) $U(t, s) P(s)=P(t) U(t, s), t \geq s \geq 0$,

(ii) the restriction $U(t, s)_{\mid}$: $\operatorname{Ker} P(s) \rightarrow \operatorname{Ker} P(t), t \geq s \geq 0$, is an isomorphism, and we denote its inverse by $U(s, t)_{\mid}:=\left(U(t, s)_{\mid}\right)^{-1}, 0 \leq s \leq t$,

(iii) $\|U(t, s) x\| \leq N e^{-\nu(t-s)}\|x\|$ for $x \in P(s) X, t \geq s \geq 0$,

(iv) $\|U(s, t) \mid x\| \leq N e^{-\nu(t-s)}\|x\|$ for $x \in \operatorname{Ker} P(t), t \geq s \geq 0$.

The projections $P(t), t \geq 0$, are called the dichotomy projections, and the constants $N, \nu$ are the dichotomy constants.

Note that the exponential dichotomy of $\{U(t, s)\}_{t \geq s \geq 0}$ implies that $H:=\sup _{t \geq 0}\|P(t)\|$ $<\infty$ and the map $t \mapsto P(t)$ is strongly continuous (see [20, Lemma 4.2]). We can then define the Green's function on the half-line as follows:

$$
\mathcal{G}(t, \tau)= \begin{cases}P(t) U(t, \tau) & \text { for } t>\tau \geq 0 \\ -U(t, \tau)_{\mid}(I-P(\tau)) & \text { for } 0 \leq t<\tau .\end{cases}
$$

It follows from the exponential dichotomy of $\{U(t, s)\}_{t \geq s \geq 0}$ that

$$
\|\mathcal{G}(t, \tau)\| \leq N(1+H) e^{-\nu|t-\tau|} \quad \text { for all } t \neq \tau, t, \tau \in \mathbb{R} .
$$

Next, using the projections $(P(t))_{t \geq 0}$ on $X$, we can define the family of operators $(\widetilde{P}(t))_{t \geq 0}$ on $\mathcal{C}$ as follows:

$$
\widetilde{P}(t): \mathcal{C} \rightarrow \mathcal{C}, \quad(\widetilde{P}(t) \phi)(\theta)=U(t-\theta, t) P(t) \phi(0) \quad \text { for all } \theta \in[-r, 0] .
$$

Then, we have that $(\widetilde{P}(t))^{2}=\widetilde{P}(t)$, and therefore the operators $\widetilde{P}(t), t \geq 0$, are projections on $\mathcal{C}$. Moreover,

$$
\operatorname{Im} \widetilde{P}(t)=\left\{\phi \in \mathcal{C}: \phi(\theta)=U(t-\theta, t) \nu_{0}, \forall \theta \in[-r, 0] \text { for some } \nu_{0} \in \operatorname{Im} P(t)\right\} .
$$

To obtain the existence of invariant stable manifolds we also need the following notion of the $\varphi$-Lipschitz of the nonlinear delay term $\Phi$.

Definition 2.2 ( $\varphi$-Lipschitz functions). Let $E$ be an admissible Banach function space and $\varphi$ be a positive function belonging to $E$. A function $\Phi:[0, \infty) \times \mathcal{C} \rightarrow X$ is said to be $\varphi$-Lipschitz if $\Phi$ satisfies

(i) $\|\Phi(t, 0)\| \leq \varphi(t)$ for all $t \in \mathbb{R}_{+}$, 
(ii) $\left\|\Phi\left(t, \phi_{1}\right)-\Phi\left(t, \phi_{2}\right)\right\| \leq \varphi(t)\left\|\phi_{1}-\phi_{2}\right\|_{\mathcal{C}}$ for all $t \in \mathbb{R}_{+}$and all $\phi_{1}, \phi_{2} \in \mathcal{C}$.

For the reader's convenience we now collect all the hypotheses on $F, B(t)$, and $\Phi$ in the following assumption.

Standing Hypothesis 2.3. We assume the following hypotheses on $F, B(t)$, and $\Phi$.

(1) The family of operators $(B(t))_{t \geq 0}$ generates an evolution family $(U(t, s))_{t \geq s \geq 0}$ as defined in Definition 1.1. Furthermore, the domain of each $B(t)$ is independent of $t$, and is denoted by $D(B)$ which is a Banach space with norm $\|\cdot\|_{D(B)}$ such that $\|B(t) x\| \leq \widetilde{K}\|x\|_{D(B)}$ for all $x \in D(B)$.

(2) The difference operator $F: \mathcal{C} \rightarrow D(B)$ is of the form $F \phi=\phi(0)-\Psi \phi$ for all $\phi \in \mathcal{C}$ where $\Psi \in \mathcal{L}(\mathcal{C}, D(B))$ satisfies $\|\Psi\|<1$.

(3) The delay operator $\Phi$ is $\varphi$-Lipschitz as defined in Definition 2.2 .

Note that if $\Phi(t, \phi)$ is $\varphi$-Lipschitz, then $\|\Phi(t, \phi)\| \leq \varphi(t)\left(1+\|\phi\|_{\mathcal{C}}\right)$ for all $\phi \in \mathcal{C}$ and $t \geq 0$. Using the operators $F, B(t)$, and $\Phi$ we can now define the operator

$$
\widetilde{\Phi}: \mathbb{R}_{+} \times C([-r, \infty), D(B)) \times \mathcal{C} \rightarrow X
$$

by

$$
\widetilde{\Phi}(t, v, \phi)=-B(t) F v_{t}+B(t) v(t)+\Phi(t, \phi) .
$$

Then, the operator $\widetilde{\Phi}$ satisfies

(i) $\|\widetilde{\Phi}(t, 0,0)\| \leq \varphi(t)$ for all $t \in \mathbb{R}_{+}$,

(ii) $\|\widetilde{\Phi}(t, u, \phi)-\widetilde{\Phi}(t, v, \psi)\| \leq \widetilde{K}\|\Psi\|\left\|u_{t}-v_{t}\right\|_{\mathcal{C}}+\varphi(t)\|\phi-\psi\|_{\mathcal{C}}$

for all $t \in \mathbb{R}_{+}, \phi, \psi \in \mathcal{C}$ and all $u, v \in C([-r, \infty), D(B))$.

We next rewrite the equation (1.1) in the form

$$
\left\{\begin{array}{l}
\frac{\partial}{\partial t} F u_{t}=B(t) F u_{t}+\widetilde{\Phi}\left(t, u, u_{t}\right) \quad t \in(0, \infty), \\
u_{0}=\phi \in \mathcal{C}:=C([-r, 0], X) .
\end{array}\right.
$$

In the space of infinite dimension, instead of (2.4) we consider the following integral equation

(2.5) $F u_{t}=U(t, 0) F u_{0}+\int_{0}^{t} U(t, \xi) \widetilde{\Phi}\left(\xi, u, u_{\xi}\right) d \xi \quad$ for all $t>0$, herewith given $u_{0} \in \mathcal{C}$.

We note that, if the evolution family $\{U(t, s)\}_{t \geq s \geq 0}$ is generated by $(B(t))_{t>0}$ (as in the situation of well-posed Cauchy problem $(1.2)$, then the function $u:[-r, \infty) \rightarrow X$, which 
satisfies 2.5 for some given function $\phi$, is called a mild solution of the semi-linear problem 2.4. The reader is referred to $\mathrm{J}$. Wu [22] for detailed treatments on the relations between classical and mild solutions of functional evolution equations. Also, the direct calculations yield that the function $u$ satisfies 2.5 if and only if it satisfies

$$
F u_{t}=U(t, s) F u_{s}+\int_{s}^{t} U(t, \xi) \widetilde{\Phi}\left(\xi, u, u_{\xi}\right) d \xi \quad \text { for all } t>s \geq 0 \text {, herewith } u_{s} \in \mathcal{C} .
$$

We now give the notion of an invariant stable manifold for the solutions of the integral equation (2.5) in the next definition.

Definition 2.4. A set $S \subset \mathbb{R}_{+} \times \mathcal{C}$ is said to be an invariant stable manifold of $\mathcal{E}$-class for the solutions to 2.5 if for every $t \in \mathbb{R}_{+}$the phase spaces $\mathcal{C}$ splits into a direct sum $\mathcal{C}=\operatorname{Im} \widetilde{P}(t) \oplus \operatorname{Ker} \widetilde{P}(t)$ with corresponding projections $\widetilde{P}(t)$ and there exists a family of Lipschitz continuous mappings

$$
\widetilde{y}_{t}: \operatorname{Im} \widetilde{P}(t) \rightarrow \operatorname{Ker} \widetilde{P}(t), \quad t \in \mathbb{R}_{+}
$$

with the Lipschitz constants independent of $t$ such that

(i) $S=\left\{\left(t, \psi+\widetilde{y}_{t}(\psi)\right) \in \mathbb{R}_{+} \times(\operatorname{Im} \widetilde{P}(t) \oplus \operatorname{Ker} \widetilde{P}(t)) \mid t \in \mathbb{R}_{+}, \psi \in \widetilde{X}_{0}(t)\right\}$, and we denote by

$$
S_{t}:=\left\{\psi+\widetilde{y}_{t}(\psi):\left(t, \psi+\widetilde{y}_{t}(\psi)\right) \in S\right\},
$$

(ii) $S_{t}$ is homeomorphic to $\operatorname{Im} \widetilde{P}(t)$ for all $t \geq 0$,

(iii) to each $\phi \in S_{s}$ there corresponds one and only one solution $u(t)$ to $(2.6)$ on $[s-r, \infty)$ satisfying the conditions that $\widetilde{u}_{s}=\phi$, and the function $\chi_{[s, \infty)}(t) u_{t}, t \in \mathbb{R}$, belongs to $\mathcal{E} \cap \mathcal{E}_{\infty}$ where the function $\widetilde{u}_{s}$ is defined by $\widetilde{u}_{s}(\theta)=F u_{s-\theta}$ for all $-r \leq \theta \leq 0$.

(iv) $S$ is positively $F$-invariant under 2.5 in the sense that if $u(t), t \geq s-r$, is a solution to 2.6 satisfying conditions that $\widetilde{u}_{s} \in S_{s}$ and the function $\chi_{[s, \infty)}(t) u_{t}$, $t \in \mathbb{R}$, belongs to $\mathcal{E}$, then we have $\widetilde{u}_{t} \in S_{t}$ for all $t \geq s$, where the function $\widetilde{u}_{t}$ is defined by

$$
\widetilde{u}_{t}(\theta)=F u_{t-\theta} \quad \text { for all }-r \leq \theta \leq 0 \text { and } t \geq 0 .
$$

Note that if we denoted by $\widetilde{X}_{0}(t)=\operatorname{Im} \widetilde{P}(t), \widetilde{X}_{1}(t)=\operatorname{Ker} \widetilde{P}(t)$ and we identify $\widetilde{X}_{0}(t) \oplus$ $\widetilde{X}_{1}(t)$ with $\widetilde{X}_{0}(t) \times \widetilde{X}_{1}(t)$, then we can write $S_{t}=\operatorname{graph}\left(\widetilde{y}_{t}\right)$.

The following lemma gives the form of bounded solutions to 2.5 . To do this, we first recall the notion of the integral translation operators $\Lambda_{1}, \Lambda_{\nu}^{\prime}, \Lambda_{\nu}^{\prime \prime}$ (see 8, Definition 2.4, Proposition 2.7]) as follows: for $\varphi \in E, \Lambda_{1} \varphi$ is defined by $\Lambda_{1} \varphi(t):=\int_{t}^{t+1} \varphi(\tau) d \tau$ belong to $E$ for all $t \in \mathbb{R}_{+}$; if $\varphi \in L_{1, \text { loc }}(\mathbb{R})$ such that $\varphi \geq 0$ and $\Lambda_{1} \varphi \in E ; \nu>0$ then $\Lambda_{\nu}^{\prime}, \Lambda_{\nu}^{\prime \prime}$ are defined by $\Lambda_{\nu}^{\prime} \varphi(t)=\int_{0}^{t} e^{-\nu(t-s)} \varphi(s) d s ; \Lambda_{\nu}^{\prime \prime} \varphi(t)=\int_{t}^{\infty} e^{-\nu(s-t)} \varphi(s) d s$ belong to $E$. 
Lemma 2.5. Assume Standing Hypothesis 1.3 and let $\varphi \in E^{\prime}$ be an exponentially Einvariant function defined as in that Standing Hypothesis. Suppose that $F,(B(t))_{t>0}$, and $\Phi$ satisfy Standing Hypothesis 2.3 and let $\widetilde{\Phi}$ be defined as in (2.3). Let the evolution family $\{U(t, s)\}_{t \geq s \geq 0}$ generated by $(B(t))_{t>0}$ have an exponential dichotomy with the dichotomy projections $P(t), t \geq 0$, and constants $N, \nu>0$. Suppose that $u(t)$ is a solution to (2.6) such that, for fixed $s \geq 0$ the function $\chi_{[s, \infty)}(t) u_{t}, t \in \mathbb{R}$, belongs to $\mathcal{E} \cap \mathcal{E}_{\infty}$. Then, for $t \geq s$ the function $u(t)$ satisfies

$$
F u_{t}=U(t, s) \nu_{0}+\int_{s}^{\infty} \mathcal{G}(t, \tau) \widetilde{\Phi}\left(\tau, u, u_{\tau}\right) d \tau
$$

for some $\nu_{0} \in X_{0}(s)=P(s) X$, where $\mathcal{G}(t, \tau)$ is the Green's function defined as in (2.1).

Proof. Put

$$
y(t)= \begin{cases}\int_{s}^{\infty} \mathcal{G}(t, \tau) \widetilde{\Phi}\left(\tau, u, u_{\tau}\right) d \tau & \text { for } t \geq s \\ \int_{s}^{\infty} \mathcal{G}(2 s-t, \tau) \widetilde{\Phi}\left(\tau, u, u_{\tau}\right) d \tau & \text { for } s-r \leq t<s\end{cases}
$$

We have, for $t \geq s$

$$
\begin{aligned}
\|y(t)\| \leq & \int_{s}^{\infty} N(1+H) e^{-\nu|t-\tau|}\left[\widetilde{K}\|\Psi\|\left\|u_{\tau}\right\|_{\mathcal{C}}+\varphi(\tau)\left(1+\left\|u_{\tau}\right\|_{\mathcal{C}}\right)\right] d \tau \\
\leq & N \widetilde{K}(1+H)\|\Psi\| \sup _{\xi \geq s-r}\|u(\xi)\| \int_{s}^{\infty} e^{-\nu|t-\tau|} d \tau \\
& +N(1+H)\left(1+\sup _{\xi \geq s-r}\|u(\xi)\|\right) \int_{s}^{\infty} e^{-\nu|t-\tau|} \varphi(\tau) d \tau \\
\leq & \frac{2}{\nu} N \widetilde{K}(1+H)\|\Psi\| \sup _{\xi \geq s-r}\|u(\xi)\| \\
& +N(1+H)\left(1+\sup _{\xi \geq s-r}\|u(\xi)\|\right) \int_{0}^{\infty} e^{-\nu|t-\tau|} \varphi(\tau) d \tau
\end{aligned}
$$

and, for $s-r \leq t<s$

$$
\begin{aligned}
\|y(t)\| \leq & \int_{s}^{\infty} N(1+H) e^{-\nu|2 s-t-\tau|}\left[\widetilde{K}\|\Psi\|\left\|u_{\tau}\right\|_{\mathcal{C}}+\varphi(\tau)\left(1+\left\|u_{\tau}\right\|_{\mathcal{C}}\right)\right] d \tau \\
\leq & \frac{2}{\nu} N \widetilde{K}(1+H)\|\Psi\| \sup _{\xi \geq s-r}\|u(\xi)\| \\
& +N(1+H)\left(1+\sup _{\xi \geq s-r}\|u(\xi)\|\right) \int_{0}^{\infty} e^{-\nu|2 s-t-\tau|} \varphi(\tau) d \tau .
\end{aligned}
$$

Since $t+\theta \in[-r+t, t]$ for fixed $t \in[s, \infty)$ and all $r \in[-r, 0]$, we have that

$$
\left\|y_{t}\right\|_{\mathcal{C}}=\sup _{\theta \in[-r, 0]} y(t+\theta) \leq \frac{2}{\nu} N \widetilde{K}(1+H)\|\Psi\| \sup _{t \geq s}\|u(t)\|
$$




$$
\begin{aligned}
& +N(1+H)\left(1+\sup _{t \geq s}\|u(t)\|\right) e^{\nu r} \int_{0}^{\infty} e^{-\nu|t-\tau|} \varphi(\tau) d \tau \\
\leq & \frac{2}{\nu} N \widetilde{K}(1+H)\|\Psi\| \sup _{t \geq s}\|u(t)\| \\
& +N(1+H)\left(1+\sup _{t \geq s}\|u(t)\|\right) e^{\nu r}\left(\Lambda_{\nu}^{\prime} \varphi(t)+\Lambda_{\nu}^{\prime \prime} \varphi(t)\right) \quad \text { for } t \geq s .
\end{aligned}
$$

Therefore, by Banach lattice properties we have that $y(\cdot) \in \mathcal{E}_{\infty}$ and

$$
\begin{aligned}
\|y(\cdot)\|_{\mathcal{E}_{\infty}} \leq & \frac{2}{\nu} N \widetilde{K}(1+H)\|\Psi\| \sup _{t \geq s}\|u(t)\| \\
& +N(1+H)\left(1+\sup _{t \geq s}\|u(t)\|\right) e^{\nu r} \frac{\left(N_{1}\left\|\Lambda_{1} T_{1}^{+} \varphi\right\|_{E}+N_{2}\left\|\Lambda_{1} \varphi\right\|_{E}\right)}{1-e^{-\nu}}
\end{aligned}
$$

where $T_{1}^{+}$is defined as in [8, Definition 2.4].

On the other hand,

$$
\begin{aligned}
U(t, s) y(s)= & -\int_{s}^{t} U(t, s) U(s, \tau)_{\mid}(I-P(\tau)) \widetilde{\Phi}\left(\tau, u, u_{\tau}\right) d \tau \\
& -\int_{t}^{\infty} U(t, s) U(s, \tau)_{\mid}(I-P(\tau)) \widetilde{\Phi}\left(\tau, u, u_{\tau}\right) d \tau \\
= & -\int_{s}^{t} U(t, \tau)(I-P(\tau)) \widetilde{\Phi}\left(\tau, u, u_{\tau}\right) d \tau \\
& -\int_{t}^{\infty} U(t, \tau)_{\mid}(I-P(\tau)) \widetilde{\Phi}\left(\tau, u, u_{\tau}\right) d \tau
\end{aligned}
$$

Therefore,

$$
y(t)=U(t, s) y(s)+\int_{s}^{t} U(t, \tau) \widetilde{\Phi}\left(\tau, u, u_{\tau}\right) d \tau .
$$

Since $u_{t}$ is a solution of (2.5) we obtain that $F u_{t}-y(t)=U(t, s)\left(F u_{s}-y(s)\right)$. Put now $\nu_{0}=F u_{s}-y(s)$. The boundedness of $F u_{t}$ and $y(t)$ on $[s, \infty)$ implies that $\nu_{0} \in X_{0}(s)$ and $P(s) F u_{s}=\nu_{0}$. Therefore, $F u_{t}=U(t, s) \nu_{0}+y(t)$ for $t \geq s$.

Remark 2.6. Equation 2.8 is called the Lyapunov-Perron's equation. By computing directly, we can see that the converse of Lemma 2.5 is also true. This means that, all solutions of the integral equation (2.8) satisfy (2.6) for $t \geq s$.

We come to our next result on the existence and partially stability of solutions starting from a subspace of $\mathcal{C}$.

Theorem 2.7. Assume Standing Hypothesis 1.3 and let $\varphi \in E^{\prime}$ be an exponentially Einvariant function and $h_{\nu}$ be defined as in that Standing Hypothesis. Suppose that F, $(B(t))_{t>0}$, and $\Phi$ satisfy Standing Hypothesis 2.3 with $(B(t))_{t>0}$ generating an evolution 
family $(U(t, s))_{t \geq s \geq 0}$ having an exponential dichotomy. Let $\widetilde{P}(t), t \geq 0$, be projections defined as in 2.2, and set

$$
\begin{aligned}
k:= & N(1+H) e^{\nu r} \\
& \times \max \left\{\frac{2 \widetilde{K}\|\Psi\|}{\nu}+\frac{N_{1}\left\|\Lambda_{1} T_{1}^{+} \varphi\right\|_{\infty}+N_{2}\left\|\Lambda_{1} \varphi\right\|_{\infty}}{1-e^{-\nu}}, \widetilde{K}\|\Psi\|\left\|\widetilde{e}_{\nu}(\cdot)\right\|_{E}+\left\|h_{\nu}(\cdot)\right\|_{E}\right\},
\end{aligned}
$$

where $\widetilde{e}_{\nu}(t)=\left\|e^{-\nu|t-\cdot|}\right\|_{E^{\prime}}$. Then, if $k /(1-\|\Psi\|)<1$, there corresponds to each $\phi \in$ $\operatorname{Im} \widetilde{P}(s)$ one and only one solution $u(t)$ of the equation (2.8) on $[s-r, \infty)$ satisfying the conditions that $\widetilde{P}(s) \widetilde{u}_{s}=\phi$, and function $\chi_{[s, \infty)}(t) u_{t}, t \in \mathbb{R}$, belongs to $\mathcal{E} \cap \mathcal{E}_{\infty}$, where the function $\widetilde{u}_{s}$ is defined as in Definition 2.4. Moreover, the following estimate is valid for any two solutions $u(t), v(t)$ corresponding to different initial functions $\phi, \psi \in \operatorname{Im} \widetilde{P}(s)$ :

$$
\left\|u_{t}-v_{t}\right\|_{\mathcal{C}} \leq C_{\mu} e^{-\mu(t-s)}\|\phi(0)-\psi(0)\| \quad \text { for all } t \geq s \geq 0
$$

where $\mu$ is a positive number satisfying

$$
\frac{N(1+H) e^{\nu r}}{1-\|\Psi\|}\left(\frac{2 \widetilde{K}\|\Psi\|}{\nu-\mu}+\frac{N_{1}\left\|\Lambda_{1} T_{1}^{+} \varphi\right\|_{\infty}+N_{2}\left\|\Lambda_{1} \varphi\right\|_{\infty}}{1-e^{-(\nu-\mu)}}\right)<1,
$$

and

$$
C_{\mu}:=\frac{N e^{\nu r}}{1-\|\Psi\|-N(1+H) e^{\nu r}\left(\frac{2 \widetilde{K}\|\Psi\|}{\nu-\mu}+\frac{N_{1}\left\|\Lambda_{1} T_{1}^{+} \varphi\right\|_{\infty}+N_{2}\left\|\Lambda_{1} \varphi\right\|_{\infty}}{1-e^{-(\nu-\mu)}}\right)} .
$$

Proof. First, to prove that there corresponds to each $\phi \in \operatorname{Im} \widetilde{P}(s)$ one and only one solution $u(t)$ in $\mathcal{E}$ of equation 2.8 on $[s-r, \infty)$, we construct a contraction mapping. To do this, we consider from 2.2 with $\phi \in \operatorname{Im} \widetilde{P}(s)=\left\{\phi(\theta)=U(t-\theta, t) \nu_{0}:-r \leq \theta \leq 0, \nu_{0} \in \operatorname{Im} P(s)\right\}$. Clearly, $\nu_{0}=\phi(0)$.

Denote by $C_{b}([s-r, \infty), X)$ the Banach space of bounded, continuous and $X$-valued functions defined on $[s-r, \infty)$, which is endowed with the sup-norm $\|\cdot\|_{\infty}$.

We define the operator $\widetilde{\Psi}: C_{b}([s-r, \infty), X) \rightarrow C_{b}([s-r, \infty), X)$ by

$$
[\widetilde{\Psi} u](t)= \begin{cases}\Psi\left(u_{t}\right) & \text { for } s \leq t \\ \Psi\left(u_{s}\right) & \text { for } s-r \leq t \leq s\end{cases}
$$

Because $\|\Psi\|<1$ we have $\|\widetilde{\Psi}\| \leq\|\Psi\|<1$. Therefore, the operator $I-\widetilde{\Psi}$ is invertible. For $\nu_{0}=\phi(0) \in \operatorname{Im} P(s)$ as above, we define a mapping $\widetilde{F}_{\phi}: C_{b}([s-r, \infty), X) \rightarrow C_{b}([s-$ $r, \infty), X)$ by

$$
\left(\widetilde{F}_{\phi} u\right)(t)= \begin{cases}U(t, s) \nu_{0}+\int_{s}^{\infty} \mathcal{G}(t, \tau) \widetilde{\Phi}\left(\tau, u, u_{\tau}\right) d \tau & \text { for } t \geq s \\ U(2 s-t, s) \nu_{0}+\int_{s}^{\infty} \mathcal{G}(2 s-t, \tau) \widetilde{\Phi}\left(\tau, u, u_{\tau}\right) d \tau & \text { for } s-r \leq t \leq s\end{cases}
$$


We now put $T:=(I-\widetilde{\Psi})^{-1} \widetilde{F}_{\phi}$. Then, we have

$$
(T u)(t)= \begin{cases}(I-\widetilde{\Psi})^{-1}\left[U(t, s) \nu_{0}+\int_{s}^{\infty} \mathcal{G}(t, \tau) \widetilde{\Phi}\left(\tau, u, u_{\tau}\right) d \tau\right] & \text { for } t \geq s, \\ (I-\widetilde{\Psi})^{-1}\left[U(2 s-t, s) \nu_{0}+\int_{s}^{\infty} \mathcal{G}(2 s-t, \tau) \widetilde{\Phi}\left(\tau, u, u_{\tau}\right) d \tau\right] & \text { for } s-r \leq t \leq s .\end{cases}
$$

We will prove the transformation $T$ as above acts from $\mathcal{E} \cap \mathcal{E}_{\infty}$ into $\mathcal{E} \cap \mathcal{E}_{\infty}$ and is a contraction.

In fact, putting $e_{\nu}(t)=e^{-\nu|t|}$ and we have $\left(T_{s}^{+} e_{\nu}\right)(t)=e^{-\nu(t-s)}$ (see [8, Definition 2.4, Proposition 2.7])

$$
y(t)= \begin{cases}(I-\widetilde{\Psi})^{-1}\left[U(t, s) \nu_{0}+\int_{s}^{\infty} \mathcal{G}(t, \tau) \widetilde{\Phi}\left(\tau, u, u_{\tau}\right) d \tau\right] & \text { for } t \geq s, \\ (I-\widetilde{\Psi})^{-1}\left[U(2 s-t, s) \nu_{0}+\int_{s}^{\infty} \mathcal{G}(2 s-t, \tau) \widetilde{\Phi}\left(\tau, u, u_{\tau}\right) d \tau\right] & \text { for } s-r \leq t \leq s,\end{cases}
$$

then we have, for $t \geq s$

$$
\begin{aligned}
\|y(t)\| \leq \frac{1}{1-\|\widetilde{\Psi}\|}\left[N e^{-\nu(t-s)}\left\|\nu_{0}\right\|+\int_{s}^{\infty} N(1+H) e^{-\nu|t-\tau|}\left(\widetilde{K}\|\Psi\|\left\|u_{\tau}\right\|_{\mathcal{C}}+\varphi(\tau)\left(1+\left\|u_{\tau}\right\| \mathcal{C}\right)\right) d \tau\right] \\
\leq \frac{1}{1-\|\Psi\|}\left[N\left(T_{s}^{+} e_{\nu}\right)(t)\left\|\nu_{0}\right\|+\frac{2}{\nu} N \widetilde{K}(1+H)\|\Psi\| \sup _{\xi \geq s-r}\|u(\xi)\|\right. \\
\left.\quad+N(1+H)\left(1+\sup _{\xi \geq s-r}\|u(\xi)\|\right) \int_{0}^{\infty} e^{-\nu|t-\tau|} \varphi(\tau) d \tau\right],
\end{aligned}
$$

and, for $s-r \leq t \leq s$

$$
\begin{aligned}
\|y(t)\| \leq \frac{1}{1-\|\widetilde{\Psi}\|}\left[N e^{-\nu(s-t)}\left\|\nu_{0}\right\|\right. \\
\left.+\int_{s}^{\infty} N(1+H) e^{-\nu|2 s-t-\tau|}\left(\widetilde{K}\|\Psi\|\left\|u_{\tau}\right\|_{\mathcal{C}}+\varphi(\tau)\left(1+\left\|u_{\tau}\right\| \mathcal{C}\right)\right) d \tau\right] \\
\leq \frac{1}{1-\|\Psi\|}\left[N\left(T_{s}^{+} e_{\nu}\right)(t)\left\|\nu_{0}\right\|+\frac{2}{\nu} N \widetilde{K}(1+H)\|\Psi\| \sup _{\xi \geq s-r}\|u(\xi)\|\right. \\
\left.+N(1+H)\left(1+\sup _{\xi \geq s-r}\|u(\xi)\|\right) \int_{0}^{\infty} e^{-\nu|2 s-t-\tau|} \varphi(\tau) d \tau\right] .
\end{aligned}
$$

Since $t+\theta \in[-r+t, t]$ for fixed $t \in[s, \infty)$ and all $\theta \in[-r, 0]$, we have

$$
\begin{aligned}
\left\|y_{t}\right\|_{\mathcal{C}} \leq \frac{1}{1-\|\Psi\|} & {\left[N e^{\nu r}\left(T_{s}^{+} e_{\nu}\right)(t)\left\|\nu_{0}\right\|+\frac{2}{\nu} N \widetilde{K}(1+H)\|\Psi\| \sup _{t \geq s}\|u(t)\|\right.} \\
& \left.+N(1+H)\left(1+\sup _{t \geq s}\|u(t)\|\right) e^{\nu r}\left(\Lambda_{\nu}^{\prime} \varphi(t)+\Lambda_{\nu}^{\prime \prime} \varphi(t)\right)\right] \quad \text { for } t \geq s .
\end{aligned}
$$

Therefore, by Banach lattice properties we have that $y(\cdot) \in \mathcal{E}$ and

$$
\begin{aligned}
\|y(\cdot)\|_{\mathcal{E}} \leq \frac{1}{1-\|\Psi\|} & {\left[N N_{1} e^{\nu r}\left\|\nu_{0}\right\|\left\|e_{\nu}\right\|_{E}+\frac{2}{\nu} N \widetilde{K}(1+H)\|\Psi\| \sup _{t \geq s}\|u(t)\|\right.} \\
& \left.+N(1+H) e^{\nu r}\left(1+\sup _{t \geq s}\|u(t)\|\right) \frac{\left(N_{1}\left\|\Lambda_{1} T_{1}^{+} \varphi\right\|_{E}+N_{2}\left\|\Lambda_{1} \varphi\right\|_{E}\right)}{1-e^{-\nu}}\right] .
\end{aligned}
$$


Similarly, we have

$$
\begin{aligned}
\|y(\cdot)\|_{\infty} \leq \frac{1}{1-\|\Psi\|} & {\left[N e^{\nu r}\left\|\nu_{0}\right\|+\frac{2}{\nu} N \widetilde{K}(1+H)\|\Psi\| e^{\nu r} \sup _{t \geq s}\|u(t)\|\right.} \\
& \left.+N(1+H) e^{\nu r}\left(1+\sup _{t \geq s}\|u(t)\|\right) \frac{\left(N_{1}\left\|\Lambda_{1} T_{1}^{+} \varphi\right\|_{\infty}+N_{2}\left\|\Lambda_{1} \varphi\right\|_{\infty}\right)}{1-e^{-\nu}}\right] .
\end{aligned}
$$

Hence, the transformation $T$ acts from $\mathcal{E} \cap \mathcal{E}_{\infty}$ into $\mathcal{E} \cap \mathcal{E}_{\infty}$. Next, we will prove $T$ is a contraction mapping. To this purpose we first estimate

$$
\begin{aligned}
\left\|\left(\widetilde{F}_{\phi} u\right)(t)-\left(\widetilde{F}_{\phi} v\right)(t)\right\| & \leq \int_{s}^{\infty}\left\|\mathcal{G}(t, \tau)\left(\widetilde{\Phi}\left(\tau, u, u_{\tau}\right)-\widetilde{\Phi}\left(\tau, v, v_{\tau}\right)\right)\right\| d \tau \\
& \leq N(1+H) \int_{s}^{\infty} e^{-\nu|t-\tau|}\left(\widetilde{K}\|\Psi\|\left\|u_{\tau}-v_{\tau}\right\|_{\mathcal{C}}+\varphi(\tau)\left\|u_{\tau}-v_{\tau}\right\|_{\mathcal{C}}\right) d \tau \\
& \leq N(1+H)\left[\widetilde{K}\|\Psi\| \int_{0}^{\infty} e^{-\nu|t-\tau|}\left\|u_{\tau}-v_{\tau}\right\|_{\mathcal{C}} d \tau\right. \\
& \left.+\int_{0}^{\infty} e^{-\nu|t-\tau|} \varphi(\tau)\left\|u_{\tau}-v_{\tau}\right\|_{\mathcal{C}} d \tau\right] \quad \text { for } t \geq s,
\end{aligned}
$$

and similarly

$$
\begin{aligned}
& \left\|\left(\widetilde{F}_{\phi} u\right)(t)-\left(\widetilde{F}_{\phi} v\right)(t)\right\| \\
\leq & \int_{s}^{\infty}\left\|\mathcal{G}(2 s-t, \tau)\left(\widetilde{\Phi}\left(\tau, u, u_{\tau}\right)-\widetilde{\Phi}\left(\tau, v, v_{\tau}\right)\right)\right\| d \tau \\
\leq & N(1+H) \int_{s}^{\infty} e^{-\nu|2 s-t-\tau|}\left(\widetilde{K}\|\Psi\|\left\|u_{\tau}-v_{\tau}\right\|_{\mathcal{C}}+\varphi(\tau)\left\|u_{\tau}-v_{\tau}\right\|_{\mathcal{C}}\right) d \tau \\
\leq & N(1+H)\left[\widetilde{K}\|\Psi\| \int_{0}^{\infty} e^{-\nu|2 s-t-\tau|}\left\|u_{\tau}-v_{\tau}\right\|_{\mathcal{C}} d \tau\right. \\
& \left.+\int_{0}^{\infty} e^{-\nu|2 s-t-\tau|} \varphi(\tau)\left\|u_{\tau}-v_{\tau}\right\|_{\mathcal{C}} d \tau\right] \quad \text { for } s-r \leq t \leq s
\end{aligned}
$$

Using the Neumann's series, we then have

$$
\begin{aligned}
(T u)(t)-(T v)(t)= & {\left[\left(\sum_{n=0}^{\infty} \widetilde{\Psi}^{n}\right) \widetilde{F}_{\phi} u\right](t)-\left[\left(\sum_{n=0}^{\infty} \widetilde{\Psi}^{n}\right) \widetilde{F}_{\phi} v\right](t) } \\
= & {\left[\left(\widetilde{F}_{\phi} u\right)(t)-\left(\widetilde{F}_{\phi} v\right)(t)\right]+\left[\left(\widetilde{\Psi} \widetilde{F}_{\phi} u\right)(t)-\left(\widetilde{\Psi} \widetilde{F}_{\phi} v\right)(t)\right] } \\
& +\left[\left(\widetilde{\Psi}^{2} \widetilde{F}_{\phi} u\right)(t)-\left(\widetilde{\Psi}^{2} \widetilde{F}_{\phi} v\right)(t)\right]+\cdots .
\end{aligned}
$$

Next, by induction we can easily see that

$$
\begin{aligned}
\left\|\left(\widetilde{\Psi}^{n} \widetilde{F}_{\phi} u\right)(t)-\left(\widetilde{\Psi}^{n} \widetilde{F}_{\phi} v\right)(t)\right\| \\
\leq\|\Psi\|^{n} N(1+H)\left[\widetilde{K}\|\Psi\| \int_{0}^{\infty} e^{-\nu|t-\tau|}\left\|u_{\tau}-v_{\tau}\right\|_{\mathcal{C}} d \tau\right. \\
\left.+\int_{0}^{\infty} e^{-\nu|t-\tau|} \varphi(\tau)\left\|u_{\tau}-v_{\tau}\right\|_{\mathcal{C}} d \tau\right] \quad \text { for } t \geq s \text { and } n \in \mathbb{N},
\end{aligned}
$$


and, for $-r+s \leq t \leq s$ and $n \in \mathbb{N}$,

$$
\begin{aligned}
\left\|\left(\widetilde{\Psi}^{n} \widetilde{F}_{\phi} u\right)(t)-\left(\widetilde{\Psi}^{n} \widetilde{F}_{\phi} v\right)(t)\right\| \leq\|\Psi\|^{n} N(1+H)\left[\widetilde{K}\|\Psi\| \int_{0}^{\infty} e^{-\nu|2 s-t-\tau|}\left\|u_{\tau}-v_{\tau}\right\|_{\mathcal{C}} d \tau\right. \\
\left.+\int_{0}^{\infty} e^{-\nu|2 s-t-\tau|} \varphi(\tau)\left\|u_{\tau}-v_{\tau}\right\|_{\mathcal{C}} d \tau\right] .
\end{aligned}
$$

From the above claim it follows that

$$
\begin{array}{r}
\|(T u)(t)-(T v)(t)\| \leq\left(\sum_{n=0}^{\infty}\|\Psi\|^{n}\right) N(1+H)\left[\widetilde{K}\|\Psi\| \int_{0}^{\infty} e^{-\nu|t-\tau|}\left\|u_{\tau}-v_{\tau}\right\|_{\mathcal{C}} d \tau\right. \\
\left.+\int_{0}^{\infty} e^{-\nu|t-\tau|} \varphi(\tau)\left\|u_{\tau}-v_{\tau}\right\|_{\mathcal{C}} d \tau\right]
\end{array}
$$

for $t \geq s$ and $n \in \mathbb{N}$, and

$$
\begin{aligned}
\|(T u)(t)-(T v)(t)\| \leq\left(\sum_{n=0}^{\infty}\|\Psi\|^{n}\right) N(1+H)\left[\widetilde{K}\|\Psi\| \int_{0}^{\infty} e^{-\nu|2 s-t-\tau|}\left\|u_{\tau}-v_{\tau}\right\|_{\mathcal{C}} d \tau\right. \\
\left.+\int_{0}^{\infty} e^{-\nu|2 s-t-\tau|} \varphi(\tau)\left\|u_{\tau}-v_{\tau}\right\|_{\mathcal{C}} d \tau\right]
\end{aligned}
$$

for $-r+s \leq t \leq s$ and $n \in \mathbb{N}$. Since $t+\theta \in[-r+t, t]$ for fixed $t \in[s, \infty)$ and all $\theta \in[-r, 0]$ we have that,

$$
\begin{aligned}
\|(T u)(t)-(T v)(t)\|_{\mathcal{C}}= & \sup _{\theta \in[-r, 0]}\|(T u)(t+\theta)-(T v)(t+\theta)\| \\
\leq \frac{N(1+H) e^{\nu r}}{1-\|\Psi\|}\left[\tilde{K}\|\Psi\| \int_{0}^{\infty}\left(T_{\tau}^{+} e_{\nu}\right)(t)\left\|u_{\tau}-v_{\tau}\right\|_{\mathcal{C}} d \tau\right. & \\
& \left.\quad+\int_{0}^{\infty} e^{-\nu|t-\tau|} \varphi(\tau)\left\|u_{\tau}-v_{\tau}\right\|_{\mathcal{C}}\right] \quad \text { for } t \geq s .
\end{aligned}
$$

Since $\left(T^{+} e_{\nu}\right)(t) \in E^{\prime}, e^{-\nu|t-\cdot|} \varphi(\cdot) \in E^{\prime},\left\|u_{\tau}-v_{\tau}\right\|_{\mathcal{C}} \in E$, and using the "Hölder's inequality" it follows from the above inequality that

$$
\begin{aligned}
& \|(T u)(t)-(T v)(t)\|_{\mathcal{C}} \\
& \leq \frac{N(1+H) e^{\nu r}}{1-\|\Psi\|}\left[\widetilde{K}\|\Psi\|\left\|\left(T_{\cdot}^{+} e_{\nu}\right)(t)\right\|_{E^{\prime}}\left\|u_{(\cdot)}-v_{(\cdot)}\right\|_{\mathcal{C}} \|_{E}\right. \\
& \left.+\left\|e^{-\nu|t-\cdot|} \varphi(\cdot)\right\|_{E^{\prime}}\|\| u_{(\cdot)}-v_{(\cdot)}\left\|_{\mathcal{C}}\right\|_{E}\right] \\
& \leq \frac{N(1+H) e^{\nu r}}{1-\|\Psi\|}\left[\widetilde{K}\|\Psi\| \widetilde{e}_{\nu}(t)\|u(\cdot)-v(\cdot)\|_{\mathcal{E}}+h_{\nu}(t)\|u(\cdot)-v(\cdot)\|_{\mathcal{E}}\right] \quad \text { for } t \geq s
\end{aligned}
$$

According to Definition 1.2 we have

$$
\begin{aligned}
\|(T u)(\cdot)-(T v)(\cdot)\|_{\mathcal{E}} & \leq \frac{N(1+H) e^{\nu r}}{1-\|\Psi\|}\left(\widetilde{K}\|\Psi\|\left\|\widetilde{e}_{\nu}(\cdot)\right\|_{E}+\left\|h_{\nu}(\cdot)\right\|_{E}\right)\|u(\cdot)-v(\cdot)\|_{\mathcal{E}} \\
& \leq \frac{k}{1-\|\Psi\|}\|u(\cdot)-v(\cdot)\|_{\mathcal{E}}
\end{aligned}
$$


where $k$ is defined as in 2.9 . In a similar way, we have

$$
\begin{aligned}
& \|(T u)(\cdot)-(T v)(\cdot)\|_{\mathcal{E}_{\infty}} \\
\leq & \frac{N(1+H) e^{\nu r}}{1-\|\Psi\|}\left(\frac{2 \widetilde{K}\|\Psi\|}{\nu}+\frac{N_{1}\left\|\Lambda_{1} T_{1}^{+} \varphi\right\|_{\infty}+N_{2}\left\|\Lambda_{1} \varphi\right\|_{\infty}}{1-e^{-\nu}}\right)\|u(\cdot)-v(\cdot)\|_{\mathcal{E}_{\infty}} \\
\leq & \frac{k}{1-\|\Psi\|}\|u(\cdot)-v(\cdot)\|_{\mathcal{E}_{\infty}} .
\end{aligned}
$$

Therefore, if $k /(1-\|\Psi\|)<1$ the transformation $T: \mathcal{E} \cap \mathcal{E}_{\infty} \rightarrow \mathcal{E} \cap \mathcal{E}_{\infty}$ is a contraction mapping. Thus, there exists a unique $u(\cdot) \in \mathcal{E} \cap \mathcal{E}_{\infty}$ such that $T u=u$. This yields that $u(t), t \geq s-r$, is the unique solution of 2.8 with

$$
\left(\widetilde{F}_{\phi} u_{s}\right)(\theta)=U(s-\theta, s) \nu_{0}+\int_{s}^{\infty} \mathcal{G}(s-\theta, \tau) \Phi\left(\tau, u_{\tau}\right) d \tau \quad \text { for all } \theta \in[-r, 0],
$$

and $P(s) F u_{s}=\nu_{0}$. Therefore, $\widetilde{P}(s) \widetilde{u}_{s}=\phi$ by definition of $\widetilde{P}(s)$ (see Equality (2.2)).

Secondly, we now prove Inequality (2.10). Let $u(t), v(t)$ be the two solutions to (2.8) corresponding to different initial functions $\phi, \psi \in \operatorname{Im} \widetilde{P}(s)$, respectively. We have that

$$
u(t)-v(t)=(T u)(t)-(T v)(t)=\left[(I-\widetilde{\Psi})^{-1} \widetilde{F}_{\phi} u\right](t)-\left[(I-\widetilde{\Psi})^{-1} \widetilde{F}_{\psi} v\right](t) .
$$

Using Neumann's series we arrive at

$$
\begin{aligned}
u(t)-v(t)= & {\left[\left(\widetilde{F}_{\phi} u\right)(t)-\left(\widetilde{F}_{\psi} v\right)(t)\right]+\left[\left(\widetilde{\Psi}\left(\widetilde{F}_{\phi} u\right)(t)\right)-\left(\widetilde{\Psi}\left(\widetilde{F}_{\psi} v\right)(t)\right)\right] } \\
& +\left[\left(\widetilde{\Psi}^{2}\left(\widetilde{F}_{\phi} u\right)(t)\right)-\left(\widetilde{\Psi}^{2}\left(\widetilde{F}_{\psi} v\right)(t)\right)\right]+\cdots .
\end{aligned}
$$

By definition of $\widetilde{F}_{\phi}$ the norm of the first term in 2.11 can be estimated by

$$
\begin{aligned}
& \left\|\left(\widetilde{F}_{\phi} u\right)(t)-\left(\widetilde{F}_{\psi} v\right)(t)\right\| \\
\leq & N\left(T_{s}^{+} e_{\nu}\right)(t)\|\phi(0)-\psi(0)\| \\
& +N(1+H) \int_{s}^{\infty} e^{-\nu|t-\tau|}\left[\widetilde{K}\|\Psi\|\left\|u_{\tau}-v_{\tau}\right\|_{\mathcal{C}}+\varphi(\tau)\left\|u_{\tau}-v_{\tau}\right\|_{\mathcal{C}}\right] d \tau \quad \text { for } t \geq s .
\end{aligned}
$$

Again, by induction, for $t \geq s$ the norm of the $n$th term in 2.11 can be estimated by

$$
\begin{aligned}
& \left\|\left(\widetilde{\Psi}^{n} \widetilde{F}_{\phi} u\right)(t)-\left(\widetilde{\Psi}^{n} \widetilde{F}_{\psi} v\right)(t)\right\| \\
& \leq\|\Psi\|^{n}\left[N\left(T_{s}^{+} e_{\nu}\right)(t)\|\phi(0)-\psi(0)\|\right. \\
& \left.\quad+N(1+H) \int_{s}^{\infty} e^{-\nu|t-\tau|}\left[\widetilde{K}\|\Psi\|\left\|u_{\tau}-v_{\tau}\right\|_{\mathcal{C}}+\varphi(\tau)\left\|u_{\tau}-v_{\tau}\right\|_{\mathcal{C}}\right] d \tau\right]
\end{aligned}
$$

and, for $s-r \leq t \leq s$

$$
\begin{aligned}
& \left\|\left(\widetilde{\Psi}^{n} \widetilde{F}_{\phi} u\right)(t)-\left(\widetilde{\Psi}^{n} \widetilde{F}_{\psi} v\right)(t)\right\| \\
& \leq\|\Psi\|^{n}\left[N\left(T_{s}^{+} e_{\nu}\right)(t)\|\phi(0)-\psi(0)\|\right. \\
& \left.\quad+N(1+H) \int_{s}^{\infty} e^{-\nu|2 s-t-\tau|}\left[\widetilde{K}\|\Psi\|\left\|u_{\tau}-v_{\tau}\right\|_{\mathcal{C}}+\varphi(\tau)\left\|u_{\tau}-v_{\tau}\right\|_{\mathcal{C}}\right] d \tau\right] .
\end{aligned}
$$


Again, since $t+\theta \in[-r+t, t]$ for fixed $t \in[s, \infty)$ and all $\theta \in[-r, 0]$, and using (2.11) we obtain

$$
\begin{aligned}
& \left\|u_{t}-v_{t}\right\|_{\mathcal{C}} \\
& \leq \frac{1}{1-\|\Psi\|}\left[N e^{\nu r}\left(T_{s}^{+} e_{\nu}\right)(t)\|\phi(0)-\psi(0)\|\right. \\
& \left.\quad+N(1+H) e^{\nu r} \int_{s}^{\infty} e^{-\nu|t-\tau|}\left[\widetilde{K}\|\Psi\|\left\|u_{\tau}-v_{\tau}\right\|_{\mathcal{C}}+\varphi(\tau)\left\|u_{\tau}-v_{\tau}\right\|_{\mathcal{C}}\right] d \tau\right]
\end{aligned}
$$

for $t \geq s$. Put $h(t)=\left\|u_{t}-v_{t}\right\|_{\mathcal{C}}$. Then, for $t \geq s$

$$
\begin{aligned}
h(t) \leq \frac{1}{1-\|\Psi\|}[ & N e^{\nu r}\left(T_{s}^{+} e_{\nu}\right)(t)\|\phi(0)-\psi(0)\| \\
& \left.+N(1+H) e^{\nu r} \int_{s}^{\infty} e^{-\nu|t-\tau|}[\widetilde{K}\|\Psi\|+\varphi(\tau)] h(\tau) d \tau\right] .
\end{aligned}
$$

We will use the cone inequality theorem (see [13, Theorem 2.8]) applying to Banach space $E$ with the cone $\mathcal{K}$ being the set of all non-negative functions. We then consider the linear operator $A$ defined for $g \in E$ by

$$
\begin{aligned}
(A g)(t)= & \frac{N(1+H) e^{\nu r} \widetilde{K}\|\Psi\|}{1-\|\Psi\|} \int_{s}^{\infty} e^{-\nu|t-\tau|} g(\tau) d \tau \\
& +\frac{N(1+H) e^{\nu r}}{1-\|\Psi\|} \int_{s}^{\infty} e^{-\nu|t-\tau|} \varphi(\tau) g(\tau) d \tau, \quad t \geq s .
\end{aligned}
$$

By "Hölder's inequality" then

$$
\|(A g)(t)\| \leq \frac{N \widetilde{K}(1+H) e^{\nu r}\|\Psi\|}{1-\|\Psi\|} \widetilde{e}_{\nu}(t)\|g\|_{E}+\frac{N(1+H) e^{\nu r}}{1-\|\Psi\|} h_{\nu}(t)\|g\|_{E} .
$$

By the Banach lactive property of $E$, we have

$$
\|A g\|_{E} \leq \frac{N(1+H) e^{\nu r}}{1-\|\Psi\|}\left[\|\widetilde{K}\| \Psi\left\|\widetilde{e}_{\nu}\right\|_{E}+\left\|h_{\nu}\right\|_{E}\right]\|g\|_{E} .
$$

Therefore $A \in \mathcal{L}(E)$ and $\|A\| \leq k /(1-\|\Psi\|)<1$. Clearly, the cone $\mathcal{K}$ is invariant under the operator $A$. The inequality 2.12 can now be rewritten as

$$
h \leq A h+z \quad \text { for } z(t)=\frac{1}{1-\|\Psi\|}\left[N e^{\nu r}\left(T_{s}^{+} e_{\nu}\right)(t)\|\phi(0)-\psi(0)\|\right] .
$$

By the cone inequality theorem [13, Theorem 2.8] we obtain that $h \leq g$, where $g$ is a solution in $E$ of the equation $g=A g+z$ which can be rewritten as

$$
\begin{aligned}
g(t)=\frac{1}{1-\|\Psi\|}\left[N e^{\nu r}\left(T_{s}^{+} e_{\nu}\right)(t)\|\phi(0)-\psi(0)\|\right. \\
\left.+N(1+H) e^{\nu r} \int_{s}^{\infty} e^{-\nu|t-\tau|}[\widetilde{K}\|\Psi\|+\varphi(\tau)] g(\tau) d \tau\right] \quad \text { for } t \geq s \geq 0 .
\end{aligned}
$$


Next, to estimate $g$, we put $w(t)=e^{\mu(t-s)} g(t)$ for $t \geq s \geq 0$. Then, we obtain that

$$
\begin{aligned}
w(t)=\frac{1}{1-\|\Psi\|}\left[N e^{\nu r}\left(T_{s}^{+} e_{\nu-\mu}\right)(t)\|\phi(0)-\psi(0)\|\right. \\
\left.+N(1+H) e^{\nu r} \int_{s}^{\infty} e^{-\nu|t-\tau|+\mu(t-\tau)}[\widetilde{K}\|\Psi\|+\varphi(\tau)] w(\tau) d \tau\right]
\end{aligned}
$$

for $t \geq s$. We will find $w$ in $L_{\infty}[s, \infty)$ which is space of real-valued functions defined and essentially bounded on $[s, \infty)$ (endowed with the sup-norm denoted by $\|\cdot\|_{\infty}$ ). We next consider the linear operator $D$ defined on $L_{\infty}[s, \infty)$ as

$$
(D \phi)(t)=\frac{N(1+H) e^{\nu r}}{1-\|\Psi\|} \int_{s}^{\infty} e^{-\nu|t-\tau|+\mu(t-\tau)}[\widetilde{K}\|\Psi\|+\varphi(\tau)] \phi(\tau) d \tau \quad \text { for all } t \geq s .
$$

By Proposition 2.6 in [9], one can easily see that $D \in \mathcal{L}\left(L_{\infty}[s, \infty)\right)$ and

$$
\|D\| \leq \frac{N(1+H) e^{\nu r}}{1-\|\Psi\|}\left(\frac{2 \widetilde{K}\|\Psi\|}{\nu-\mu}+\frac{N_{1}\left\|\Lambda_{1} T_{1}^{+} \varphi\right\|_{\infty}+N_{2}\left\|\Lambda_{1} \varphi\right\|_{\infty}}{1-e^{-(\nu-\mu)}}\right) .
$$

The equation 2.13 can be rewritten as

$$
w=D w+\widetilde{z} \quad \text { for } \widetilde{z}(t)=\frac{1}{1-\|\Psi\|} N e^{\nu r}\left(T_{s}^{+} e_{\nu-\mu}\right)(t)\|\phi(0)-\psi(0)\| .
$$

We have $\|D\|<1$ if $\frac{N(1+H) e^{\nu r}}{1-\|\Psi\|}\left(\frac{2 \widetilde{K}\|\Psi\|}{\nu-\mu}+\frac{N_{1}\left\|\Lambda_{1} T_{1}^{+} \varphi\right\|_{\infty}+N_{2}\left\|\Lambda_{1} \varphi\right\|_{\infty}}{1-e^{-(\nu-\mu)}}\right)<1$. Under this condition, the equation $w=D w+\widetilde{z}$ has the unique solution $w \in L_{\infty}[s, \infty)$ and $w=(I-D)^{-1} \widetilde{z}$. Hence, we obtain that

$$
\begin{aligned}
\|w\|_{\infty} & =\left\|(I-D)^{-1} \widetilde{z}\right\|_{\infty} \leq \frac{N e^{\nu r}}{(1-\|D\|)(1-\|\Psi\|)}\|\phi(0)-\psi(0)\| \\
& \leq \frac{N e^{\nu r}\|\phi(0)-\psi(0)\|}{1-\|\Psi\|-N(1+H) e^{\nu r}\left(\frac{2 \widetilde{K}\|\Psi\|}{\nu-\mu}+\frac{N_{1}\left\|\Lambda_{1} T_{1}^{+} \varphi\right\|_{\infty}+N_{2}\left\|\Lambda_{1} \varphi\right\|_{\infty}}{1-e^{-(\nu-\mu)}}\right)} \\
& :=C_{\mu}\|\phi(0)-\psi(0)\| .
\end{aligned}
$$

This yields that $w(t) \leq C_{\mu}\|\phi(0)-\psi(0)\|$ for $t \geq s$. Therefore,

$$
h(t)=\left\|u_{t}-v_{t}\right\|_{\mathcal{C}} \leq g(t)=e^{-\mu(t-s)} w(t) \leq C_{\mu} e^{-\mu(t-s)}\|\phi(0)-\psi(0)\| \quad \text { for } t \geq s .
$$

We now state and prove our main result of this section.

Theorem 2.8. Assume Standing Hypothesis 1.3 and let $\varphi \in E^{\prime}$ be an exponentially Einvariant function and $h_{\nu}$ be defined as in that Standing Hypothesis. Suppose that F, $(B(t))_{t>0}$, and $\Phi$ satisfy Standing Hypothesis 2.3 with $(B(t))_{t>0}$ generating an evolution family $(U(t, s))_{t \geq s \geq 0}$ having an exponential dichotomy. Define $k$ as in (2.9) and $k_{1}$ by

$$
k_{1}:=N(1+H) e^{\nu r}\left[\frac{\widetilde{K}\|\Psi\|}{\nu}+\frac{N_{1}\left\|\Lambda_{1} T_{1}^{+} \varphi\right\|_{\infty}+N_{2}\left\|\Lambda_{1} \varphi\right\|_{\infty}}{1-e^{-\nu}}\right] .
$$


Then, if

$$
\max \left\{\frac{N k_{1} e^{\nu r}}{1-k_{1}-\|\Psi\|}, \frac{k}{1-\|\Psi\|}\right\}<1
$$

then, there exists an invariant stable manifold $S$ of $\mathcal{E}$-class for the solutions to (2.5).

Moreover, every two solutions $u(t), v(t)$ on the manifold $S$ of $\mathcal{E}$-class for the solutions to (2.5) corresponding to different functions $\phi, \psi \in S_{s}$ attract each other exponentially in sense that, there exists positive constants $\mu$ and $C_{\mu}$ independent of $s \geq 0$ such that

$$
\left\|u_{t}-v_{t}\right\|_{\mathcal{C}} \leq C_{\mu} e^{-\mu(t-s)}\|\widetilde{P}(s) \phi-\widetilde{P}(s) \psi\|_{\mathcal{C}} \quad \text { for } t \geq s,
$$

where $\widetilde{P}(t), t \geq 0$ are defined as in 2.2 and $S_{s}$ is defined as in Definition 2.4 .

Proof. To prove the existence of an invariant stable manifold $S=\left\{\left(t, S_{t}\right)\right\}_{t \geq 0}$ of $\mathcal{E}$-class for the solution to (2.5) satisfies the conditions of Definition 2.4. We have, since $\{U(t, s)\}_{t \geq s \geq 0}$ has an exponential dichotomy, and for each $t \geq 0$ the phase space $\mathcal{C}$ splits into the direct $\operatorname{sum} \mathcal{C}=\operatorname{Im} \widetilde{P}(t) \oplus \operatorname{Ker} \widetilde{P}(t)$ where the projections $\widetilde{P}(t), t \geq 0$, are defined as in $(2.2)$. We determine the surface $S_{t}$ for $t \geq 0$ by the formula

$$
S_{t}:=\left\{\phi+\widetilde{y}_{t}(\phi): \phi \in \operatorname{Im} \widetilde{P}(t)\right\} \subset \mathcal{C}
$$

where the operator $\widetilde{y}_{t_{0}}$ is defined for each $t_{0} \geq 0$ by

$$
\widetilde{y}_{t_{0}}(\phi)(\theta)=\int_{t_{0}}^{\infty} \mathcal{G}\left(t_{0}-\theta, \tau\right) \widetilde{\Phi}\left(\tau, u, u_{\tau}\right) d \tau \quad \text { for all } \theta \in[-r, 0],
$$

here $u(\cdot)$ is the unique solution of $(2.5)$ on $\left[-r+t_{0}, \infty\right)$ satisfying $\widetilde{P}\left(t_{0}\right) u_{t_{0}}=\phi$ (note that the existence and uniqueness of $u(\cdot)$ is guaranteed by Theorem 2.7). On the other hand, by the definition of the Green's function $\mathcal{G}$ (see (2.1)) we have that $\widetilde{y}_{t_{0}}(\phi) \in \operatorname{Ker} \widetilde{P}\left(t_{0}\right)$. We next show that the stable manifold $S$ satisfies the conditions of Definition 2.4.

First, we prove that $\widetilde{y}_{t_{0}}$ is Lipschitz continuity with Lipschitz constant independent of $t_{0}$. Indeed, for $\phi$ and $\psi$ belonging to $\operatorname{Im} \widetilde{P}\left(t_{0}\right)$ we have

$$
\begin{aligned}
& \left\|\widetilde{y}_{t_{0}}(\phi)(\theta)-\widetilde{y}_{t_{0}}(\psi)(\theta)\right\| \\
\leq & N(1+H) \int_{t_{0}}^{\infty} e^{-\nu\left|t_{0}-\theta-\tau\right|}[\widetilde{K}\|\Psi\|+\varphi(\tau)]\left\|u_{\tau}-v_{\tau}\right\|_{\mathcal{C}} d \tau \\
\leq & N(1+H) e^{\nu r} \int_{t_{0}}^{\infty} e^{-\nu\left|t_{0}-\tau\right|}[\widetilde{K}\|\Psi\|+\varphi(\tau)]\left\|u_{\tau}-v_{\tau}\right\|_{\mathcal{C}} d \tau \\
\leq & N(1+H) e^{\nu r} \sup _{\tau \geq t_{0}}\left\|u_{\tau}-v_{\tau}\right\|_{\mathcal{C}} \int_{t_{0}}^{\infty} e^{-\nu\left|t_{0}-\tau\right|}[\widetilde{K}\|\Psi\|+\varphi(\tau)] d \tau \\
\leq & N(1+H) e^{\nu r}\left[\frac{\widetilde{K}\|\Psi\|}{\nu}+\frac{N_{1}\left\|\Lambda_{1} T_{1}^{+} \varphi\right\|_{\infty}+N_{2}\left\|\Lambda_{1} \varphi\right\|_{\infty}}{1-e^{-\nu}}\right] \sup _{\tau \geq t_{0}}\left\|u_{\tau}-v_{\tau}\right\|_{\mathcal{C}} .
\end{aligned}
$$


Moreover, by the Lyapunov-Perron's equation for $u(\cdot)$ and $v(\cdot)$, for $\tau \geq t_{0}$ we have

$$
\begin{aligned}
& \sup _{\tau \geq t_{0}}\left\|u_{\tau}-v_{\tau}\right\|_{\mathcal{C}} \\
& \leq \frac{1}{1-\|\Psi\|}\left[N e^{\nu r}\|\phi-\psi\|_{\mathcal{C}}\right. \\
& \left.\quad+N(1+H) e^{\nu r}\left(\frac{\widetilde{K}\|\Psi\|}{\nu}+\frac{N_{1}\left\|\Lambda_{1} T_{1}^{+} \varphi\right\|_{\infty}+N_{2}\left\|\Lambda_{1} \varphi\right\|_{\infty}}{1-e^{-\nu}}\right) \sup _{\tau \geq t_{0}}\left\|u_{\tau}-v_{\tau}\right\|_{\mathcal{C}}\right] .
\end{aligned}
$$

It follows that

$$
\sup _{\tau \geq t_{0}}\left\|u_{\tau}-v_{\tau}\right\|_{\mathcal{C}} \leq \frac{N e^{\nu r}}{1-k_{1}-\|\Psi\|}\|\phi-\psi\|_{\mathcal{C}}
$$

Substituting this inequality to $(2.15)$ we obtain that

$$
\left\|\widetilde{y}_{t_{0}}(\phi)-\widetilde{y}_{t_{0}}(\psi)\right\|_{\mathcal{C}}=\sup _{\theta \in[-r, 0]}\left\|\widetilde{y}_{t_{0}}(\phi)(\theta)-\widetilde{y}_{t_{0}}(\psi)(\theta)\right\| \leq \frac{N k_{1} e^{\nu r}}{1-k_{1}-\|\Psi\|}\|\phi-\psi\|_{\mathcal{C}} .
$$

Therefore, $\widetilde{y}_{t_{0}}$ is Lipschitz continuous with the Lipschitz constant $N k_{1} e^{\nu r} /\left(1-k_{1}-\|\Psi\|\right)$ independent of $t_{0}$.

To show $S_{t_{0}}$ is homeomorphic to $\operatorname{Im} \widetilde{P}\left(t_{0}\right)$, we define the transformation $\mathbf{D}: \operatorname{Im} \widetilde{P}\left(t_{0}\right)$ $\rightarrow S_{t_{0}}$ by $\mathbf{D} \phi:=\phi+\widetilde{y}_{t_{0}}(\phi)$ for all $\phi \in \operatorname{Im} \widetilde{P}\left(t_{0}\right)$. Then, applying the Implicit Function Theorem for Lipschitz continuous mappings (see [21, Lemma 2.7]) we have that if the Lipschitz constant $N^{2} N_{1}(1+H) e^{2 \nu r}\left\|e_{\nu}\right\|_{E}\|\varphi\|_{E^{\prime}} /(1-k-\|\Psi\|)<1$ (equivalently $k<$ $\left.1-\|\Psi\|-N^{2} N_{1}(1+H) e^{2 \nu r}\left\|e_{\nu}\right\|_{E}\|\varphi\|_{E^{\prime}}\right)$ then $\mathbf{D}$ is a homeomorphism. Therefore, the condition (ii) in Definition 2.4 is satisfied.

The condition (iii) in Definition 2.4 now follows from Theorem 2.7. We now prove that the condition (iv) of Definition 2.4 is satisfied. Indeed, let $u(\cdot)$ be solution of (2.5) such that the function $u_{s} \in S_{s}$. Then, by Lemma 2.5, for $t \in[s, \infty)$ the solution $u(t)$ satisfies

$$
F u_{t}=U(t, s) \nu_{0}+\int_{s}^{\infty} \mathcal{G}(t, \tau) \widetilde{\Phi}\left(\tau, u, u_{\tau}\right) d \tau \quad \text { for some } \nu_{0} \in \operatorname{Im} P(s) .
$$

Thus, for $t \geq s$ and $\theta \in[-r, 0]$ we have

$$
\begin{aligned}
F u_{t-\theta}= & U(t-\theta, s) \nu_{0}+\int_{s}^{\infty} \mathcal{G}(t-\theta, \tau) \widetilde{\Phi}\left(\tau, u, u_{\tau}\right) d \tau \\
= & U(t-\theta, s) \nu_{0}+\int_{s}^{t} \mathcal{G}(t-\theta, \tau) \widetilde{\Phi}\left(\tau, u, u_{\tau}\right) d \tau+\int_{t}^{\infty} \mathcal{G}(t-\theta, \tau) \widetilde{\Phi}\left(\tau, u, u_{\tau}\right) d \tau \\
= & U(t-\theta, s) \nu_{0}+\int_{s}^{t} U(t-\theta, \tau) P(\tau) \widetilde{\Phi}\left(\tau, u, u_{\tau}\right) d \tau+\int_{t}^{\infty} \mathcal{G}(t-\theta, \tau) \widetilde{\Phi}\left(\tau, u, u_{\tau}\right) d \tau \\
= & U(t-\theta, t)\left[U(t, s) \nu_{0}+\int_{s}^{t} U(t, \tau) P(\tau) \widetilde{\Phi}\left(\tau, u, u_{\tau}\right) d \tau\right] \\
& +\int_{t}^{\infty} \mathcal{G}(t-\theta, \tau) \widetilde{\Phi}\left(\tau, u, u_{\tau}\right) d \tau \quad \text { for all }-r \leq \theta \leq 0 .
\end{aligned}
$$


Put $z_{0}=U(t, s) \nu_{0}+\int_{s}^{t} U(t, \tau) P(\tau) \widetilde{\Phi}\left(\tau, u, u_{\tau}\right) d \tau$. We have $P(t) z_{0}=z_{0}$, hence $z_{0} \in$ $\operatorname{Im} P(t)$. We thus obtain that the function $\phi(\theta):=U(t-\theta, t) z_{0},-r \leq \theta \leq 0$, belongs to $\operatorname{Im} \widetilde{P}(t)$ and

$$
F u_{t-\theta}=U(t-\theta, t) z_{0}+\int_{t}^{\infty} \mathcal{G}(t-\theta, \tau) \widetilde{\Phi}\left(\tau, u, u_{\tau}\right) d \tau \quad \text { for all }-r \leq \theta \leq 0 .
$$

By the uniqueness of $u(\cdot)$ on $[s-r, \infty)$ as in the proof of Theorem 2.7 we have that (2.5) has a unique solution $u(\cdot)$ on $[-r+t, \infty)$ satisfying $\widetilde{P}(t) u_{t}=\phi$ and

$$
F u_{\xi}=U(2 t-\xi, t) z_{0}+\int_{t}^{\infty} \mathcal{G}(2 \xi-t, \tau) \widetilde{\Phi}\left(\tau, u, u_{\tau}\right) d \tau
$$

for $\xi \in[-r+t, t]$. Therefore, for $t \geq s$ the function $\widetilde{u}_{t}$ defined as in 2.7 satisfies

$$
\begin{aligned}
\widetilde{u}_{t}(\theta) & =F u_{t-\theta}=U(t-\theta, t) z_{0}+\int_{t}^{\infty} \mathcal{G}(t-\theta, \tau) \widetilde{\Phi}\left(\tau, u, u_{\tau}\right) d \tau \\
& =\phi(\theta)+\widetilde{y}_{t}(\phi)(\theta) \quad \text { for all }-r \leq \theta \leq 0
\end{aligned}
$$

where, as seen above, $\phi \in \operatorname{Im} \widetilde{P}(t)$. Hence, $\widetilde{u}_{t} \in S_{t}$ for $t \geq s$.

Finally, the inequality (2.14) follows from inequality 2.10 in Theorem 2.7.

We illustrate our result by the following example.

Example 2.9. Consider the following neutral partial functional differential equation

$$
\begin{aligned}
\frac{\partial w(x, t)}{\partial t}-l \frac{\partial w(x, t-1)}{\partial t}= & a(t)\left[\frac{\partial^{2} w(x, t)}{\partial x^{2}}+\alpha w(x, t)\right] \\
& +b t e^{-\alpha t} \int_{-1}^{0} \ln (1+|w(x, t+\theta)|) d \theta \quad \text { for } 0 \leq x \leq \pi, t \geq 0, \\
w(0, t)= & w(\pi, t)=0 \text { for } t \geq 0, \\
w_{s}(x, \theta)= & w(x, s+\theta) \text { for all } x \in[0, \pi], \theta \in[-1,0],
\end{aligned}
$$

where $l$ and $\alpha$ are real constants with $|l|<1, \alpha>1$ and $\alpha \neq n^{2}, \forall n \in \mathbb{N}$. The function $a(\cdot) \in L_{1, \text { loc }}\left(\mathbb{R}_{+}\right)$and satisfies the condition $\gamma_{1} \geq a(t) \geq \gamma_{0}>0$ for fixed constants $\gamma_{0}$, $\gamma_{1}$ and a.e. $t \geq 0$. We choose the Hilbert space $X:=L_{2}[0, \pi], \mathcal{C}:=C([-1,0], X)$ and let $B: D(B) \subset X \rightarrow X$ be defined by

$$
B(f)=f^{\prime \prime}+\alpha f
$$

with the domain $D(B)=H_{0}^{2}[0, \pi]:=\left\{f \in W^{2,2}[0, \pi]: f(0)=f(\pi)=0\right\}$.

Also define the difference and delay operators $F$ and $\Phi$ as

$$
\begin{aligned}
& F: \mathcal{C} \rightarrow D(B), \quad F(f):=f(0)-l f(-1), \\
& \Phi: \mathbb{R}_{+} \times \mathcal{C} \rightarrow X, \quad \Phi(t, \phi):=b t e^{-\alpha t} \int_{-1}^{0} \ln (1+|(\phi(\theta))(x)|) d \theta, \quad x \in[0, \pi] .
\end{aligned}
$$


Note that the fact that $\Phi$ takes value in $X=L_{2}[0, \pi]$ can be easily seen by using the Minkowski's inequality.

Putting now $B(t):=a(t) B$ the equation $(2.16)$ can now be rewritten as

$$
\left\{\begin{array}{l}
\frac{\partial}{\partial t} F u_{t}(\cdot)=B(t) u(t)+\Phi\left(t, u_{t}(\cdot, \theta)\right) \quad t \geq s \geq 0 \\
u_{s}(\cdot, \theta)=\phi(\cdot, \theta) \in \mathcal{C}
\end{array}\right.
$$

where $B$ is the generator of an analytic semigroup $(T(t))_{t>0}$, with $\sigma(B)=\{-1+\alpha,-4+$ $\alpha, \ldots\}$. Since $\alpha \neq n^{2}, \forall n \in \mathbb{N}$, we have that $\sigma(B) \cap i \mathbb{R}=\emptyset$. Applying the spectral mapping theorem for analytic semigroups we get

$$
\sigma(T(t))=e^{t \sigma(B)}=\left\{e^{t(\alpha-1)}, e^{t(\alpha-4)}, \ldots\right\}
$$

and $\sigma(T(t)) \cap\{z \in \mathbb{C}:|z|=1\}=\emptyset$ for all $t>0$. Therefore, for fixed $t_{0}$, the spectrum of the operator $T\left(t_{0}\right)$ splits into two disjoint sets $\sigma_{0}, \sigma_{1}$, where $\sigma_{0} \subset\{z \in \mathbb{C}:|z|<1\}, \sigma_{1} \subset$ $\{z \in \mathbb{C}:|z|>1\}$. Next, we choose $P=P\left(t_{0}\right)$ to be the Riesz projections corresponding to the spectral set $\sigma_{0}$, and $Q=I-P$. Clearly, $P$ and $Q$ commute with $T(t)$ for all $t>0$. We denote by $T_{Q}(t):=T(t) Q$ the restriction of $T(t)$ on $\operatorname{Im} Q$. As well-known in Semigroup Theory, in this case, the semigroup $(T(t))_{t>0}$ is called hyperbolic (or having an exponential dichotomy) and the restriction $T_{Q}(t)$ is invertible. Moreover, there are positive constants $N, \gamma$ such that

$$
\begin{aligned}
\left\|\left.T(t)\right|_{P X}\right\| & \leq N e^{-\gamma t}, & & \forall t \geq 0, \\
\left\|T_{Q}(-t)\right\| & =\left\|T_{Q}(t)^{-1}\right\| \leq N e^{-\gamma t}, & & \forall t \geq 0 .
\end{aligned}
$$

Clearly, the family $(B(t))_{t \geq 0}=(a(t) B)_{t \geq 0}$ generates the evolution family $(U(t, s))_{t \geq s \geq 0}$ defined by the formula:

$$
U(t, s)=T\left(\int_{s}^{t} a(\tau) d \tau\right) .
$$

From the dichotomy estimates of $(T(t))_{t>0}$ in 2.18 , it is straightforward to check that the evolution family $(U(t, s))_{t \geq s \geq 0}$ has an exponential dichotomy with projections $P$ and constants $N, \nu:=\gamma \gamma_{0}$ by the following estimates:

$$
\begin{aligned}
\left\|\left.U(t, s)\right|_{P X}\right\| & =\left\|\left.T\left(\int_{s}^{t} a(\tau) d \tau\right)\right|_{P X}\right\| \leq N e^{-\nu(t-s)}, \\
\left\|U(s, t)_{\mid}\right\| & =\left\|\left(\left.U(t, s)\right|_{\operatorname{Ker} P}\right)^{-1}\right\|=\left\|T_{Q}\left(-\int_{s}^{t} a(\tau) d \tau\right)\right\| \leq N e^{-\nu(t-s)}
\end{aligned}
$$

for all $t \geq s \geq 0$.

Clearly, the difference operator $F$ be of form $F=\delta_{0}-\Psi$ for $\Psi=l \delta_{-1}$ and $\|\Psi\| \leq|l|<1$. The linear operator $B(t)$ for every fixed $t \geq 0$ with norm $\|\cdot\|_{H_{0}^{2}[0, \pi]}$ and $\|B(t) u\| \leq$ 
$\widetilde{K}\|u\|_{H_{0}^{2}[0, \pi]}$. We now take $E=L_{p}\left(\mathbb{R}_{+}\right) 1 \leq p \leq+\infty$, the delay operator $\Phi: \mathbb{R}_{+} \times \mathcal{C} \rightarrow X$ defined as in (2.17) and check that $\Phi$ is $\varphi$-Lipschitz with $\varphi(t)=|b| t e^{-\alpha t} \in E=L_{p}\left(\mathbb{R}_{+}\right)$, $p \geq 1$. Indeed, the condition (i) in Definition 2.2 is evident. To verify the condition (ii) in that definition we use Minkowski's inequality and the fact that $\ln (1+h) \leq h$ for all $h \geq 0$. Then,

$$
\begin{aligned}
& \left\|\Phi\left(t, \phi_{1}\right)(x)-\Phi\left(t, \phi_{2}\right)(x)\right\|_{2} \\
= & |b| t e^{-\alpha t}\left(\int_{0}^{\pi}\left(\int_{-1}^{0} \ln \frac{1+\left|\left(\phi_{1}(\theta)\right)(x)\right|}{1+\left|\left(\phi_{2}(\theta)\right)(x)\right|} d \theta\right)^{2} d x\right)^{1 / 2} \\
\leq & |b| t e^{-\alpha t} \int_{-1}^{0}\left(\int_{0}^{\pi} \ln ^{2} \frac{1+\left|\left(\phi_{1}(\theta)\right)(x)\right|}{1+\left|\left(\phi_{2}(\theta)\right)(x)\right|} d x\right)^{1 / 2} d \theta \\
= & |b| t e^{-\alpha t} \int_{-1}^{0}\left(\int_{0}^{\pi} \ln ^{2}\left(1+\frac{\left|\left(\phi_{1}(\theta)\right)(x)\right|-\left|\left(\phi_{2}(\theta)\right)(x)\right|}{1+\left|\left(\phi_{2}(\theta)\right)(x)\right|} d x\right)^{1 / 2} d \theta\right. \\
\leq & |b| t e^{-\alpha t} \int_{-1}^{0}\left(\int_{0}^{\pi}\left|\left(\phi_{1}(\theta)\right)(x)-\left(\phi_{2}(\theta)\right)(x)\right|^{2} d x\right)^{1 / 2} d \theta \\
= & |b| t e^{-\alpha t} \int_{-1}^{0}\left\|\phi_{1}(\theta)-\phi_{2}(\theta)\right\|_{2} d \theta \\
\leq & |b| t e^{-\alpha t} \sup _{\theta \in[-1,0]}\left\|\phi_{1}(\theta)-\phi_{2}(\theta)\right\|_{2} .
\end{aligned}
$$

Hence, $\Phi$ is $\varphi$-Lipschitz. In the space $L_{p}\left(\mathbb{R}_{+}\right)$, the constants $N_{1}, N_{2}$ are defined by $N_{1}=N_{2}=1$,

$$
\Lambda_{1} \varphi(t)=\int_{t}^{t+1} \varphi(\tau) d \tau \quad \text { and } \quad \Lambda_{1} T_{1}^{+} \varphi(t)=\int_{(t-1)_{+}}^{t} \varphi(\tau) d \tau
$$

where $(t-1)_{+}=\max \{0, t-1\}$. Thus,

$$
\max \left\{\left\|\Lambda_{1} \varphi\right\|_{\infty},\left\|\Lambda_{1} T_{1}^{+} \varphi\right\|_{\infty}\right\}<\frac{|b|\left(1+e^{-1}-e^{-\alpha}\right)}{\alpha^{2}} .
$$

By Theorem 2.8 we obtain that if

$$
\frac{|b|\left(1+e^{-1}-e^{-\alpha}\right)}{\alpha^{2}}<\frac{\left(1-e^{-\nu}\right)}{2}\left[\frac{(1-\|\Psi\|) e^{-\nu}}{N(1+H)\left(1+N e^{\nu}\right)}-\frac{\tilde{K}\|\Psi\|}{\nu}\right]
$$

then there is an invariant stable manifold $S$ of $L_{p}$-class for the mild solutions to the problem 2.16.

3. Exponential trichotomy and center-stable manifolds

In this section, we will generalize Theorem 2.8 to the case that the evolution family $\{U(t, s)\}_{t \geq s \geq 0}$ has an exponential trichotomy on $\mathbb{R}_{+}$and the nonlinear forcing term $\Phi$ is 
$\varphi$-Lipschitz. In this case, under similar conditions as in above section we will prove that there exists a center-invariant stable manifold of $\mathcal{E}$-class for the solutions to (2.5). We now recall the definition of an exponential trichotomy and a center-invariant stable manifold of $\mathcal{E}$-class.

Definition 3.1. A given evolution family $\{U(t, s)\}_{t \geq s \geq 0}$ is said to have an exponential trichotomy on the half-line if there are three families of projections $\left\{P_{j}(t)\right\}, t \geq 0, j=$ $1,2,3$, and positive constants $N, \alpha, \beta$ with $\alpha<\beta$ such that the following conditions are fulfilled:

(i) $\sup _{t \geq 0}\left\|P_{j}(t)\right\|<\infty, j=1,2,3$.

(ii) $P_{1}(t)+P_{2}(t)+P_{3}(t)=$ Id for $t \geq 0$ and $P_{j}(t) P_{i}(t)=0$ for all $j \neq i$.

(iii) $P_{j}(t) U(t, s)=U(t, s) P_{j}(s)$ for $t \geq s \geq 0$ and $j=1,2,3$.

(iv) $\left.U(t, s)\right|_{\operatorname{Im} P_{j}(s)}$ are homeomorphisms from $\operatorname{Im} P_{j}(s)$ onto $\operatorname{Im} P_{j}(t)$, for all $t \geq s \geq 0$ and $j=2,3$, respectively; we also denote the inverse of $\left.U(t, s)\right|_{\operatorname{Im} P_{j}(s)}$ by $U(s, t)$, $0 \leq s \leq t$

(v) For all $t \geq s \geq 0$ and $x \in X$, the following estimates hold:

$$
\begin{aligned}
\left\|U(t, s) P_{1}(s) x\right\| & \leq N e^{-\beta(t-s)}\left\|P_{1}(s) x\right\|, \\
\left\|U(s, t){ }_{\mid} P_{2}(t) x\right\| & \leq N e^{-\beta(t-s)}\left\|P_{2}(t) x\right\|, \\
\left\|U(t, s) P_{3}(s) x\right\| & \leq N e^{\alpha(t-s)}\left\|P_{3}(s) x\right\| .
\end{aligned}
$$

The projections $\left\{P_{j}(t)\right\}, t \geq 0, j=1,2,3$, are called the trichotomy projections, and the constants $N, \alpha, \beta$ are the trichotomy constants.

Using the trichotomy projections we can now construct three families of projections $\left\{\widetilde{P}_{j}(t)\right\}, t \geq 0, j=1,2,3$, on $\mathcal{C}$ as follows:

$$
\left(\widetilde{P}_{j}(t) \phi\right)(\theta)=U(t-\theta, t) P_{j}(t) \phi(0) \quad \text { for all } \theta \in[-r, 0] \text { and } \phi \in \mathcal{C} .
$$

Definition 3.2. Let the evolution family $\{U(t, s)\}_{t \geq s \geq 0}$ have an exponential trichotomy with the trichotomy projections $\left\{P_{j}(t)\right\} t \geq 0, j=1,2,3$, and constants $N, \alpha, \beta$ given as in Definition 3.1 .

A set $S \subset \mathbb{R}_{+} \times \mathcal{C}$ is said to be a center-invariant stable manifold of $\mathcal{E}$-class for the solutions to 2.5 if there exists a family of Lipschitz continuous mappings

$$
\Phi_{t}: \operatorname{Im}\left(\widetilde{P}_{1}(t)+\widetilde{P}_{3}(t)\right) \rightarrow \operatorname{Im} \widetilde{P}_{2}(t)
$$

with projections $\left\{\widetilde{P}_{j}(t)\right\}, t \geq 0, j=1,2,3$ defined as in 3.1), and Lipschitz constants being independent of $t$ such that $S_{t}=\operatorname{graph}\left(\Phi_{t}\right)$ has the following properties: 
(i) $S_{t}$ is homeomorphic to $\operatorname{Im}\left(\widetilde{P}_{1}(t)+\widetilde{P}_{3}(t)\right)$ for all $t \geq 0$.

(ii) To each $\phi \in S_{s}$ there corresponds one and only one solution $u(t)$ to 2.5$)$ on $[s-r, \infty)$ satisfying $e^{-\gamma(s+\theta)} F u_{s-\theta}=\phi(\theta)$ for $\theta \in[-r, 0]$ and

$$
z_{t}=\left\{\begin{array}{ll}
e^{-\gamma(t+\cdot)} u_{t}(\cdot) & \text { for } t \geq s \geq 0, \\
0 & \text { for } t<s
\end{array} \quad \text { belong to } \mathcal{E}, \text { where } \gamma=\frac{\beta+\alpha}{2} .\right.
$$

Moreover, for any two solutions $u(t)$ and $v(t)$ to 2.5 corresponding to different functions $\phi, \psi \in S_{s}$ we have the estimate

$$
\left\|u_{t}-v_{t}\right\|_{\mathcal{C}} \leq C_{\mu} e^{(\gamma-\mu)(t-s)}\|(\widetilde{P}(s) \phi)(0)-(\widetilde{P}(s) \psi)(0)\| \quad \text { for } t \geq s,
$$

where $\mu, C_{\mu}$ are positive constants independent of $s, u(\cdot)$, and $v(\cdot)$ and $\widetilde{P}(t)=$ $\widetilde{P}_{1}(t)+\widetilde{P}_{3}(t)$.

(iii) $S$ is positively $F$-invariant under 2.5) in the sense that, if $u(t), t \geq s-r$, is the solution to 2.5 satisfying the conditions that the function $e^{-\gamma(s+\cdot)} \widetilde{u}_{s}(\cdot) \in S_{s}$ and

$$
z_{t}=\left\{\begin{array}{ll}
e^{-\gamma(t+\cdot)} u_{t}(\cdot) & \text { for } t \geq s \geq 0, \\
0 & \text { for } t<s
\end{array} \quad \text { belong to } \mathcal{E},\right.
$$

then the function $e^{-\gamma(t+\cdot)} \widetilde{u}_{t}(\cdot) \in S_{t}$ for all $t \geq s$ where $\widetilde{u}_{t}$ is defined as in (2.7).

We come to our second main result. It proves the existence of a center-stable manifold for solutions of (2.5).

Theorem 3.3. Let the evolution family $\{U(t, s)\}_{t \geq s \geq 0}$ have an exponential trichotomy with the trichotomy projections $\left\{P_{j}(t)\right\}, t \geq 0, j=1,2,3$, and constants $N, \alpha, \beta$ given as in Definition 3.1. Suppose that $\Phi: \mathbb{R}_{+} \times \mathcal{C} \rightarrow X$ is $\varphi$-Lipschitz, where $\varphi$ is a positive function which belongs to $E$. Set $q:=\sup \left\{\left\|P_{j}(t)\right\|: t \geq 0, j=1,3\right\}, N_{0}:=\max \{N, 2 N q\}$, $\nu=(\beta-\alpha) / 2$ and

$$
k_{2}:=N_{0}(1+H) e^{\nu r}\left[\frac{\tilde{K}\|\Psi\|}{\nu}+\frac{N_{1}\left\|\Lambda_{1} T_{1}^{+} \varphi\right\|_{\infty}+N_{2}\left\|\Lambda_{1} \varphi\right\|_{\infty}}{1-e^{-\nu}}\right] .
$$

Then, if $k_{2}<(1-\|\Psi\|) /\left(1+N_{0} e^{\nu r}\right)$, for each fixed $\beta>\alpha$ there exists a center-invariant stable manifold of $\mathcal{E}$-class for the solutions to 2.5 .

Proof. Set $P(t):=P_{1}(t)+P_{3}(t)$ and $Q(t):=P_{2}(t)=\mathrm{Id}-P(t)$ for $t \geq 0$. We have that $P(t)$ and $Q(t)$ are projections complemented to each other on $X$. We then define the families of projections $\left\{\widetilde{P}_{j}(t)\right\}, t \geq 0, j=1,2,3$, on $\mathcal{C}$ as in Equality $(3.1)$. Setting $\widetilde{P}(t)=\widetilde{P}_{1}(t)+\widetilde{P}_{3}(t)$ 
and $\widetilde{Q}(t)=\widetilde{P}_{2}(t), t \geq 0$, we obtain that $\widetilde{P}(t)$ and $\widetilde{Q}(t)$ are complemented projections on $\mathcal{C}$ for each $t \geq 0$. We consider the following rescaling evolution family

$$
\widetilde{U}(t, s)=e^{-\gamma(t-s)} U(t, s) \text { for all } t \geq s \geq 0, \text { where } \gamma:=\frac{\beta+\alpha}{2} .
$$

We now prove that the evolution family $\widetilde{U}(t, s)$ has an exponential dichotomy with dichotomy projections $P(t), t \geq 0$. Indeed,

$$
\begin{aligned}
P(t) \widetilde{U}(t, s) & =e^{-\gamma(t-s)}\left(P_{1}(t)+P_{3}(t)\right) U(t, s) \\
& =e^{-\gamma(t-s)} U(t, s)\left(P_{1}(s)+P_{3}(s)\right)=\widetilde{U}(t, s) P(s) .
\end{aligned}
$$

Since $\left.U(t, s)\right|_{\operatorname{Im} P_{2}(s)}$ is a homeomorphism from $\operatorname{Im} P_{2}(s)$ onto $\operatorname{Im} P_{2}(t)$ and $\operatorname{Im} P_{2}(t)=$ Ker $P(t)$ for all $t \geq 0$, we have that $\left.\widetilde{U}(t, s)\right|_{\text {Ker } P(s)}$ is also a homeomorphism from $\operatorname{Ker} P(s)$ onto Ker $P(t)$, and we denote $\widetilde{U}(s, t)_{\mid}:=\left(\left.\widetilde{U}(t, s)\right|_{\operatorname{Ker} P(s)}\right)^{-1}$ for $0 \leq s \leq t$. By the definition of exponential trichotomy we have

$$
\|\widetilde{U}(s, t) \mid Q(t) x\| \leq e^{-(\beta+\gamma)(t-s)}\|Q(t) x\| \quad \text { for all } t \geq s \geq 0 .
$$

On the other hand,

$$
\begin{aligned}
\|\widetilde{U}(t, s) P(s) x\| & =e^{-\gamma(t-s)}\left\|U(t, s)\left(P_{1}(s)+P_{3}(s)\right) x\right\| \\
& \leq N e^{-\gamma(t-s)}\left(e^{-\beta(t-s)}\left\|P_{1}(s) x\right\|+e^{\alpha(t-s)}\left\|P_{3}(s) x\right\|\right) \\
& =N e^{-\gamma(t-s)}\left(e^{-\beta(t-s)}\left\|P_{1}(s) P(s) x\right\|+e^{\alpha(t-s)}\left\|P_{3}(s) P(s) x\right\|\right)
\end{aligned}
$$

for all $t \geq s \geq 0$ and $x \in X$.

Putting $q:=\sup \left\{\left\|P_{j}(t)\right\|: t \geq 0, j=1,3\right\}$, we finally get the following estimate

$$
\|\widetilde{U}(t, s) P(s) x\| \leq 2 N q e^{-\frac{(\beta-\alpha)}{2}(t-s)}\|P(s) x\| .
$$

Therefore, $\widetilde{U}(t, s)$ has an exponential dichotomy with the dichotomy projections $P(t)$, $t \geq 0$, and constants $N_{0}:=\max \{N, 2 N q\}, \nu:=(\beta-\alpha) / 2$. Put $\widehat{u}(t)=e^{-\gamma t} u(t)$, and define the mapping $\Phi^{*}: \mathbb{R}_{+} \times \mathcal{C} \rightarrow X$ as follows:

$$
\Phi^{*}(t, \phi)=e^{-\gamma t} \Phi\left(t, e^{\gamma(t+\cdot)} \phi(\cdot)\right) \quad \text { for }(t, \phi) \in \mathbb{R}_{+} \times \mathcal{C} .
$$

Obviously, $\Phi^{*}$ is also $\varphi$-Lipschitz. Therefore, we can also define the operator $\widetilde{\Phi}^{*}: \mathbb{R}_{+} \times$ $\mathcal{C} \times D(B) \rightarrow X$ by

$$
\widetilde{\Phi}^{*}(t, \phi, x)=-B(t) F x_{t}+B(t) x(t)+\Phi^{*}(t, \phi) .
$$

Thus, we can rewrite 2.5 in the new form

$$
\begin{aligned}
F \widehat{u}_{t} & =\widetilde{U}(t, s) F \widehat{u}_{s}+\int_{s}^{t} \widetilde{U}(t, \xi) \widetilde{\Phi}^{*}\left(\xi, \widehat{u}, \widehat{u}_{\xi}\right) d \xi \quad \text { for all } t \geq s \geq 0, \\
\widehat{u}_{s}(\cdot) & =e^{-\gamma(s+\cdot)} \phi(\cdot) \in \mathcal{C} .
\end{aligned}
$$


Hence, by Theorem 2.8, we obtain that, if $k_{2}<(1-\|\Psi\|) /\left(1+N_{0} e^{\nu r}\right)$, then there exists an invariant stable manifold of $\mathcal{E}$-class $S$ for the solutions to 3.2 . Returning to (2.5) by using the relation $u(t):=e^{\gamma t} \widehat{u}(t)$ and Theorems 2.7 and 2.8, we can easily verify the properties of $S$ which are stated in (i), (ii) and (iii) in Definition 3.2. Thus, $S$ is a center-invariant stable manifold of $\mathcal{E}$-class for the solutions of $(2.5)$.

\section{Acknowledgments}

We would like to thank the referee very much for his/her careful reading of the manuscript. His/her corrections and suggestions lead to the improvement of the paper. This research is funded by Vietnam National Foundation for Science and Technology Development (NAFOSTED) under Grant number 101.02-2017.303.

\section{References}

[1] R. Benkhalti, K. Ezzinbi and S. Fatajou, Stable and unstable manifolds for nonlinear partial neutral functional differential equations, Differential Integral Equations 23 (2010), no. 7-8, 773-794.

[2] N. N. Bogoliubov and Y. A. Mitropolsky, Asymptotic Methods in the Theory of Nonlinear Oscillations, Translated from the second revised Russian edition, International Monographs on Advanced Mathematics and Physics Hindustan, Gordon and Breach Science Publishers, New York, 1961.

[3] - The method of integral manifolds in nonlinear mechanics, Contributions to Differential Equations 2 (1963), 123-196.

[4] J. L. Dalec'kiu and M. G. Kreŭn, Stability of Solutions of Differential Equations in Banach Spaces, Translations of Mathematical Monographs 43, American Mathematical Society, Provindence, R.I., 1974.

[5] J. Hadamard, Sur l'itération et les solutions asymptotiques des équations différentielles, Bull. Soc. Math. France 29 (1901), 224-228.

[6] D. Henry, Geometric Theory of Semilinear Parabolic Equations, Lecture Notes in Mathematics 840, Springer-Verlag, Berlin, 1981.

[7] N. T. Huy, Exponential dichotomy of evolution equations and admissibility of function spaces on a half-line, J. Funct. Anal. 235 (2006), no. 1, 330-354.

[8] _ Invariant manifolds of admissible classes for semi-linear evolution equations, J. Differential Equations 246 (2009), no. 5, 1820-1844. 
[9] N. T. Huy and P. V. Bang, Invariant stable manifolds for partial neutral functional differential equations in admissible spaces on A half-line, Discrete Contin. Dyn. Syst. Ser. B 20 (2015), no. 9, 2993-3011.

[10] N. T. Huy and V. T. N. Ha, Admissibly integral manifolds for semilinear evolution equations, Ann. Polon. Math. 112 (2014), no. 2, 127-163.

[11] J. L. Massera and J. J. Schäffer, Linear Differential Equations and Function Spaces, Pure and Applied Mathematics 21, Academic Press, New York, 1966.

[12] R. Nagel and G. Nickel, Well-posedness for nonautonomous abstract Cauchy problems, in: Evolution Equations, Semigroups and Functional Analysis (Milano, 2000), 279293, Progr. Nonlinear Differential Equations Appl. 50, Birkhäuser, Basel, 2002.

[13] T. H. Nguyen, Stable manifolds for semi-linear evolution equations and admissibility of function spaces on a half-line, J. Math. Anal. Appl. 354 (2009), no. 1, 372-386.

[14] T. H. Nguyen and V. D. Trinh, Integral manifolds for partial functional differential equations in admissible spaces on a half-line, J. Math. Anal. Appl. 411 (2014), no. 2, $816-828$.

[15] A. Pazy, Semigroups of Linear Operators and Applications to Partial Differential Equations, Applied Mathematical Sciences 44, Springer-Verlag, Berlin, 1983.

[16] O. Perron, Über Stabilität und asymptotisches Verhalten der Integrale von Differentialgleichungssystemen, (German) Math. Z. 29 (1929), no. 1, 129-160.

[17]__ Die Stabilitätsfrage bei Differentialgleichungen, (German) Math. Z. 32 (1930), no. 1, 703-728.

[18] H. Petzeltová and O. J. Staffans, Spectral decomposition and invariant manifolds for some functional partial differential equations, J. Differential Equations 138 (1997), no. $2,301-327$

[19] F. Räbiger and R. Schnaubelt, The spectral mapping theorem for evolution semigroups on spaces of vector-valued functions, Semigroup Forum 52 (1996), no. 2, 225-239.

[20] N. Van Minh, F. Räbiger and R. Schnaubelt, Exponential stability, exponential expansiveness, and exponential dichotomy of evolution equations on the half-line, Integral Equations Operator Theory 32 (1998), no. 3, 332-353.

[21] N. Van Minh and J. Wu, Invariant manifolds of partial functional differential equations, J. Differential Equations 198 (2004), no. 2, 381-421. 
[22] J. Wu, Theory and Applications of Partial Functional-differential Equations, Applied Mathematical Sciences 119, Springer-Verlag, New York, 1996.

Thieu Huy Nguyen

School of Applied Mathematics and Informatics, Hanoi University of Science and Technology, 1 Dai Co Viet, Hanoi, Vietnam

E-mail address: huy.nguyenthieu@hust.edu.vn

Xuan Yen Trinh

Department of Basic Sciences, Hung Yen University of Technology and Education, Khoai Chau, Dan Tien, Hung Yen, Vietnam

E-mail address: trinhyenspkt@gmail.com 